\title{
EXPERIMENTAL DATA FROM ANALYSIS OF SEQUENTIAL PRECIPITATION SAMPLES AT BROOKHAVEN NATIONAL LABORATORY
}

\author{
Gilbert S. Raynor and Janet V. Hayes
}

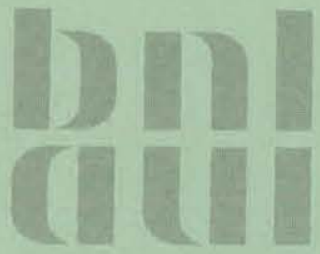

April 1978



BROOKHAVEN NATIONAL LABORATORY ASSOCIATED UNIVERSITIES, INC. UNDER CONTRACT NO. EY-76-C-02-0016 WITH THE UNITED STATES DEPARTMENT OF ENERGY 


\section{DISCLAIMER}

This report was prepared as an account of work sponsored by an agency of the United States Government. Neither the United States Government nor any agency Thereof, nor any of their employees, makes any warranty, express or implied, or assumes any legal liability or responsibility for the accuracy, completeness, or usefulness of any information, apparatus, product, or process disclosed, or represents that its use would not infringe privately owned rights. Reference herein to any specific commercial product, process, or service by trade name, trademark, manufacturer, or otherwise does not necessarily constitute or imply its endorsement, recommendation, or favoring by the United States Government or any agency thereof. The views and opinions of authors expressed herein do not necessarily state or reflect those of the United States Government or any agency thereof. 


\section{DISCLAIMER}

Portions of this document may be illegible in electronic image products. Images are produced from the best available original document. 


\section{EXPERIMENTAL DATA FROM ANALYSIS OF SEQUENTIAL PRECIPITATION SAMPLES AT BROOKHAVEN NATIONAL LABORATORY}

Gilbert S. Raynor and Janet V. Hayes

April 1978

This report was prepared as an account of work sponsored by the United States Government. Neither the United States nor the United States Department of Energy, nor any of their employees, nor any of their contractors, subcontractors, or their employees, makes any werranty. express ar implied, or assumues any leat liability or responsibility for the accuracy, completeness or usefulness of any information, apparitus product or process disclosed, ar represents that its use would 


\section{NOTICE}

This report was prepared as an account of work spuisored by the United States Government. Neither the United States nor the United States Department of Energy (DOE), nor any of their employees, nor any of their contractors, subcontractors, or their employees, makes any warranty, express or implied, or assumes any legal liability or responsibility for the accuracy, completeness or usefulness of any information, apparatus, product or process disclosed, or represents that its use would not infringe privately owned rights.

Printed in the United States of America

Available from

National Technical Information Service

U.S. Department of Commerce

5285 Port Royal Road

Springfield, VA 22161

Price: Printed Copy $\$ 4.50$; Microfiche $\$ 3.00$

May 1978

360 copies 


\section{ABSTRACT}

Hcurly precipitation samples were collected at Brookhaven National Laboratory from June 1976 through May 1977 using an Automatic Sequential Precipitation Sampler. Conventional meteorological data were recorded for each sample period. The samples were analyzed for $\mathrm{pH}$, conductivity and concentrations of sulfate, nitrate, ammonium, sodium and chloride ions. Statistical analyses were performed of the relationships between selected chemical varlables and between them and meteorological conditions. Results are presented in a series of tables. 
INTRODUCTION

This report contains results of statistical analyses of the chemistry of hourly precipitation samples collected at Brookhaven National Laboratory from June 1, 1976 to May 31, 1977, and the relationships between the chemical variables and selected meteorological parameters. Nearly all hours having precipitation amounts large enough for analysis are included in the data base. Only two precipitation events and small portions of two others were missed due to mechanical problems or to outages caused by lightning or power failure.

The data are presented here for the benefit of other users. Interpretation of the data will be presented elsewhere.

\section{EXPERIMENTAL METHODS}

Precipitation samples were taken with the Brookhaven Automatic Sequential Precipitation Sampler. A description of the sampler, its operation and method of handling the samples were presented earlier. (1) Samples were analyzed by the Analytical Chemistry Group, Department of Energy and Environment, using procedures described as follows:

$\mathrm{pH}$ is determined as soon as possible after the sample is recelved using an Orion pH meter.

SPECIFIC CONDUCTIVITY is determined as soon as possible after the sample is received using a Markson Electromark Digital Analyzer. 
$\mathrm{NO}_{3}^{-}+\mathrm{NO}_{2}^{-}$are determined colorimetrically using an automated procedure and the Technicon Auto Analyzer. $\mathrm{NO}_{3}{ }^{-}$is reduced with hydrazine and the $\mathrm{NO}_{2}{ }^{-}$reacts with sulfanilamide under acidic conditions to form a diazo compound. This compound couples with N-I-naphthylethylenediamine dihydrochloride to form a reddish-purple azo dye which is read at $530 \mathrm{~nm}$. Samples are refrigerated prior to analysis to prevent degradation of the ions.

$\mathrm{NH}_{4}^{+}$is determined using the Berthelot reaction in which a blue-colored compound, indophenol blue, is formed when a solution of an ammonium salt is added to sodium phenoxide followed by the addition of sodium hypochlorite in an alkaline medium. The indophenol blue is measured at $630 \mathrm{~nm}$. The procedure is automated and run on a Technicon Auto Analyzer. Samples are refrigerated prior to this analysis to prevent degradation of the $\mathrm{NH}_{4}^{+}$.

$\mathrm{SO}_{4}=$ analyses are performed using an automated turbidimetric procedure and the Technicon Auto Analyzer. $\mathrm{SO}_{4}=$ is converted to a BaSO ${ }_{4}$ suspension under controlled conditions and the resulting turbidity is read at $420 \mathrm{~nm}$. An alternate procedure occasionally used is the methyl thymol blue colorimetric procedure. Identical results are obtained with both procedures.

$\mathrm{CL}^{-}$is determined using an automated colorimetric procedure and the Pechnicon Auto Analyzer. Thiocyanate ion $\left(\mathrm{SCN}^{-}\right)$is liberated from mercuric thiocyanate through sequestering of mercury by chloride. The liberated $\mathrm{SCN}^{-}$in the presence of ferric ion forms a highly colored ferric thiocyanate, in concentration proportional to the original chloride. The complex is measured at $467 \mathrm{~nm}$. 
$\mathrm{Na}^{+}$is determined by atomic absorption analysis using a Perkin-Elmer 303 or Instrumentation Laboratory Atomic Absorption Spectrophotometer.

DATA

Data recorded for each hourly sample included date, sample number, time of beginning and end of sample, duration the cover was open, precipitation event number. within the month, precipitation amount, precipitation type, cloud type, synoptic type causing the precipitation, wind direction and speed at a height of $108 \mathrm{~m}$, air temperature and the chemical parameters enumerated above.

The meteorological parameters are se1f-explanatory except for the following cases. In precipitation type, thunderstorm hours include all hours in which a thunderstorm occurred even though the thunderstorm did not last the entire hour. It also includes hours of thunderstorm with hail. Snow includes all frozen precipitation, such. as sleet and snow showers since not enough hours of these types occurred to classify them separately.

Under synoptic type, low includes all cases in which the precipitation could not be attributed to a specific type of front. Cold front precipitation includes precipitation both ahead of and behind the front. Squall lines include pre-frontal troughs.

Certain derived parameters were obtained from the original data. pH values were converted to molar concentrations of hydrogen ions. Ratios of selected ionic species were computed. Excess conductivity was calculated to give a measure of conductivity attributable to constituents other than $\mathrm{H}^{+}$. Excess sulfate was calculated to determine the non-seawater 
sulfate contribution using the sulfate/chloride and chloride/sodium ratios in seawater. Sodium was used as the reference since the chloride/ sodium ratios in the precipitation samples were consistently above the seawater ratio suggesting sources of chloride other than the ocean.

\section{DATA ANALYSIS}

Analytical procedures and methods of data presentation were selected so that the results would have maximum usefulness to the greater number of potential users. Statistical analyses were performed by an electronic computer making extensive use of the Statistical Package for the Social Sciences (SPSS) ${ }^{(2)}$ which eliminated much programming and facilitated computations.

Chemical parameters of the samples and ratios between them were related by standard linear correlation and regression techniques. The relationship of chemical species to meteorological conditions was determined by categorizing the chemical data by meteorological parameters and computing the mean, standard deviation and other statistics of the resulting distributions.

For these same categories, a weighted mean was computed by weighting the value of each chemical parameter by the amount of precipttation in that hour. The weighted mean values approximate those values which would be obtained if all samples taken under a selected condition were mixed and a single analysis performed. These means are most appropriate for relating precipitation chemistry to long term effects such as effects on soils or ground water. The unweighted means give greater importance to extreme events and are more appropriate for relating the chemistry of a category to short-term effects such as plant damage by "acid rain." 
All data were used except that one extreme hourly value of conductivity and one of sulfate concentration were removed for the regression analyses but not for the statistical analyses. Also, several hours of data during the peak of Hurricane Belle were removed from all analyses since they had concentrations of chloride and sodium about three orders of magnitude above normal.

\section{RESULTS}

Results are presented here in tabular form. Units are all in ppm except hydrogen ion $\left(10^{-4} \mathrm{M}\right)$ and conductivity ( $\mu$ mhos $/ \mathrm{cm}$ ). Ratios are nondimensional. Graphical presentation of selected results will be included in future analytical papers. The tables are largely self-explanatory but are described briefly below.

Relationships between chemical parameters

The relationships between selected chemical parameters are shown in Table 1 where the slope and intercept of the linear regression equations relating the variables and the linear correlation coefficient between the variables are given. Where one variable could be considered dependent and the other independent, the former was used as the y variable and the latter as the $\mathrm{x}$ variable; where no such relationship was presumed, the choice of $\mathrm{x}$ and $\mathrm{y}$ variables was arbitrary. Some pairs of variables show varying degrees of positive correlation. This does not necessarily imply a physical cause and effect since both may be related to some other variable. Other pairs show no correlation but the results are included to document that fact.

The variability of selected chemical parameters by $\mathrm{pH}$ class is shown In Tables $2 a-2 n$. Included for each class are weighted mean, mean, standard deviation, minimum, maximum and number of cases. 
Relationship of chemical to meteorological parameters

Tables $3 a-3 g$ through $18 a-18 g$ present the means and statistics of the chemical variables classified by the following meteorological variables: season, synoptic type, precipitation type, precipitation rate, wind speed, wind direction and air temperature.

\section{ACKNOWLEDGMENTS}

This study would have been impossible without the participation and cooperation of many people. Bernard Manowitz, Leonard Newman and Lance Evans participated in the original design of the project. John McNeil assisted in the design and construction of the sampler and was primarily responsible for its operation and for collection of the samples. Chemical analyses were performed under the direction of Leonard Newnan by Mary Kinsley, Daniel Leahy, Robert Wilson, Cleveland Dodge, Donna White, Tom McGee and Dan Spandau. George Hendrey, Stephen Schwartz and others suggested some of the derived parameters and supplied pertinent information from the literature. 


\section{REFERENCES}

1. Raynor, G.S. and McNeil, J.P., The Brookhaven Automatic Sequential

Precipitation Sampler. Report BNL \#50818 Brookhaven National Laboratory, Upton, New York, 29 pp. 1978.

2. Nie, N.H., Hull, C.H., Jenkins, J.G., Steinbrenner, K. and Bent, D.H. Statistical Package for the Social Sciences, 2nd ed., New York, McGrawHi11, 675 pp. 1975. 
Table 1

Correlation and Regression Analyses of Relationships

Between Chemical Variables

(See text for units.)

\begin{tabular}{|c|c|c|c|c|}
\hline Variable $y$ & Variable $\mathbf{x}$ & Intercept & Slope & Correlation Coefficient \\
\hline $\mathrm{H}^{+} \times 10^{-4}$ & $\mathrm{SO}_{4}=$ & -0.123 & 0.132 & 0.673 \\
\hline $\mathrm{H}^{+} \times 10^{-4}$ & Excess $\mathrm{SO}_{4}=$ & -0.165 & 0.160 & 0.740 \\
\hline $\mathrm{H}^{+} \times 10^{-4}$ & $\mathrm{NO}_{3}^{-}$ & 0.116 & 0.559 & 0.560 \\
\hline $\mathrm{H}^{+} \times 10^{-4}$ & $\mathrm{Cl}^{-}$ & 0.451 & -0.003 & -0.019 \\
\hline $\mathrm{H}^{+} \times 10^{-4}$ & $\mathrm{NH}_{4}^{+}$ & 0.265 & 0.366 & 0.291 \\
\hline $\mathrm{H}^{+} \times 10^{-4}$ & $\mathrm{Na}^{+}$ & 0.453 & -0.002 & -0.005 \\
\hline $\mathrm{H}^{+} \times 10^{-4}$ & $\mathrm{SO}_{4}=/ \mathrm{cl}^{-}$ & 0.093 & 0.081 & 0.491 \\
\hline $\mathrm{H}^{+} \times 10^{-4}$ & $\mathrm{SO}_{4}^{4}=/ \mathrm{Na}^{+}$ & 0.302 & 0.009 & 0.291 \\
\hline $\mathrm{H}^{+} \times 10^{-4}$ & $\mathrm{NO}_{3}-/ \mathrm{SO}_{4}=$ & 0.310 & 1.052 & 0.180 \\
\hline $\mathrm{H}^{+} \times 10^{-4}$ & $\mathrm{NH}_{4}^{+} / \mathrm{NO}_{3}^{-}$ & 0.488 & -0.030 & -0.085 \\
\hline $\mathrm{H}^{+} \times 10^{-4}$ & $\mathrm{NH}_{4}^{4}+/ \mathrm{SO}_{4}=$ & 0.463 & -0.209 & -0.031 \\
\hline $\mathrm{H}^{+} \times 10^{-4}$ & $\mathrm{Cl}^{-} / \mathrm{Na}^{+}$ & 0.487 & -0.009 & -0.049 \\
\hline Cond. & $\mathrm{H}^{+} \times 10^{-4}$ & $16.426^{\circ}$ & 28.501 & 0.692 \\
\hline Cond. & $\mathrm{SO}_{4}=$ & -1.935 & 8.09 .1 & 0.856 \\
\hline Cond. & $\mathrm{NO}_{3}^{-}$ & 11.433 & 36.166 & 0.739 \\
\hline Cond. & $\mathrm{Cl}^{-}$ & 20.843 & 3.785 & 0.532 \\
\hline Cond. & $\mathrm{NH}_{4}^{+}$ & 15.582 & 36.431 & 0.523 \\
\hline Cond. & $\mathrm{Na}^{+}$ & 20.723 & 8.603 & 0.535 \\
\hline Ex Cond. & $\mathrm{SO}_{4}=$ & 3.081 & 2.999 & 0.517 \\
\hline Ex Cond. & $\mathrm{NO}_{3}^{-}$ & 7.736 & 13.716 & 0.469 \\
\hline Ex Cond. & $\mathrm{NH}_{4}^{+}$ & 9.148 & 14.611 & 0.342 \\
\hline $\mathrm{SO}_{4}=$ & $\mathrm{NO}_{3}^{-}$ & 2.184 & 3.371 & 0.670 \\
\hline $\mathrm{SO}_{4}^{4}=$ & $\mathrm{NH}_{4}^{+}$ & 2.388 & 3.878 & 0.611 \\
\hline $\mathrm{NO}_{3}^{-}$ & $\mathrm{NH}_{4}^{4}+$ & 0.226 & 0.781 & 0.607 \\
\hline $\mathrm{Na}^{+}$ & $\mathrm{C} 1^{-}$ & -0.014 & 0.446 & 0.969 \\
\hline $\mathrm{Ex} \mathrm{SO}_{4}=$ & $\mathrm{NO}_{3}^{-}$ & 2.022 & 3.249 & 0.658 \\
\hline $\mathrm{Ex} \mathrm{SO}_{4}=$ & $\mathrm{NH}_{4}^{+}$ & 1.987 & 4.366 & 0.612 \\
\hline
\end{tabular}


Table $2 \mathrm{a}$

Variation in Conductivity $(\mu \mathrm{mhos} / \mathrm{cm})$ by $\mathrm{pH}$

\begin{tabular}{|c|c|c|c|c|c|c|}
\hline $\mathrm{pH}$ & wt. mean & mean & S. D. & min. & $\max$. & $\mathrm{n}$ \\
\hline $3.00-3.99$ & 194.32 & 109.43 & 126.03 & 38.70 & 800.00 & 35 \\
\hline $4.00-4.99$ & 21.20 & 28.03 & 20.32 & 3.20 & 163.00 & 201 \\
\hline $5.00-5.99$ & 9.15 & 12.82 & 11.12 & 2.00 & 81.20 & 104 \\
\hline $6.00-6.99$ & 15.85 & 19.33 & 13.34 & 4.60 & 52.50 & 0 \\
\hline ALL & 47.59 & 31.07 & 49.87 & 2.00 & 800.00 & 360 \\
\hline
\end{tabular}

Table 2b

Variation in Excess Conductivity ( $\mu \mathrm{mhos} / \mathrm{cm})$ by $\mathrm{pH}$

\begin{tabular}{|c|c|c|c|c|c|c|}
\hline $\mathrm{pH}$ & wt. mean & mean & S. D. & min. & $\max$. & $\mathrm{n}$ \\
\hline $3.00-3.99$ & 135.82 & 47.31 & 123.76 & 0.00 & 733.15 & 35 \\
\hline $4.00-4.99$ & 8.58 & 14.16 & 16.88 & 0.00 & 136.44 & 201 \\
\hline $5.00-5.99$ & 7.29 & 11.07 & 11.02 & 0.90 & 78.67 & 104 \\
\hline $6.00-6.99$ & 15.64 & 19.12 & 13.34 & 4.26 & 52.28 & 20 \\
\hline ALL & 30.88 & 16.76 & 41.93 & 0.00 & 733.15 & 360 \\
\hline
\end{tabular}

Table 2c

Variation in Sulfate Concentration (ppm) by $\mathrm{pH}$

\begin{tabular}{|c|c|c|c|c|c|c|}
\hline $\mathrm{pH}$ & wt. mean & mean & S. D. & min. & $\max$. & $\mathrm{n}$ \\
\hline $3.00-3.99$ & 8.04 & 10.71 & 5.92 & 2.70 & 27.00 & 49 \\
\hline $4.00-4.99$ & 2.92 & 3.72 & 2.19 & 2.00 & 24.67 & 228 \\
\hline $5.00-5.99$ & 2.39 & 3.91 & 12.56 & 1.11 & 142.50 & 126 \\
\hline $6.00-6.99$ & 2.51 & 3.59 & 2.75 & 2.00 & 13.80 & 36 \\
\hline ALL & 3.72 & 4.55 & 7.53 & 1.11 & 142.50 & 439 \\
\hline
\end{tabular}


Table 2d

Variation in Excess Sulfate Concentration ( $\mathrm{ppm}$ ) by $\mathrm{pH}$

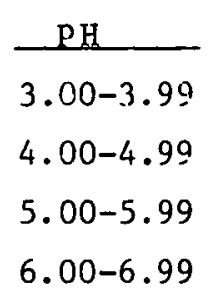

ALL

wt. mean
7.87
2.69
2.15
2.03
3.50

\begin{tabular}{r} 
mean \\
\hline 10.17 \\
3.34 \\
2.51 \\
2.88 \\
3.82
\end{tabular}

\begin{tabular}{c} 
S. D. \\
\hline 5.53 \\
2.01 \\
1.25 \\
1.77 \\
3.36
\end{tabular}

$\frac{\min }{2.78}$

$\frac{\max .}{26.96}$

$\frac{\mathrm{n}}{45}$

0.84

14.28

215

0.71

8.81

117

0.50

7.55

30

0.50

26.96

407

Table $2 \mathrm{e}$

Variation in Nitrate Concentration (ppm) by $\mathrm{pH}$

\begin{tabular}{|c|c|c|c|c|c|c|}
\hline $\mathrm{PH}$ & wt. mean & mean & S. D. & $\min$. & $\max$. & $\mathfrak{n}$ \\
\hline $3.00-3.99$ & 0.90 & 1.65 & 1.11 & 0.13 & 4.30 & 50 \\
\hline $4.00-4.99$ & 0.34 & 0.57 & 0.56 & 0.02 & 3.45 & 228 \\
\hline $5.00-5.99$ & 0.08 & 0.23 & 0.28 & 0.02 & 1.65 & 121 \\
\hline $6.00-6.99$ & 0.18 & 0.42 & 0.56 & 0.04 & 2.58 & 34 \\
\hline $7.00-7.99$ & 0.29 & 0.29 & - & 0.29 & 0.29 & 1 \\
\hline ALL & 0.36 & 0.59 & 0.72 & 0.02 & 4.30 & 434 \\
\hline
\end{tabular}

\section{Table $2 f$}

Variation in Ammonium Concentration (ppm) by $\mathrm{pH}$

\begin{tabular}{|c|c|c|c|c|c|c|}
\hline $\mathrm{pH}$ & wt. mean & mean & S. D. & min. & $\max$. & $\mathrm{n}$ \\
\hline $3.00-3.99$ & 0.58 & 0.96 & 0.70 & 0.24 & 3.50 & 48 \\
\hline $4.00-4.99$ & 0.24 & 0.38 & 0.36 & 0.05 & 3.64 & 230 \\
\hline $5.00-5.99$ & 0.11 & 0.31 & 0.43 & 0.02 & 2.58 & 125 \\
\hline $6.00-6.99$ & 0.41 & 0.90 & 1.08 & 0.08 & 5.40 & 36 \\
\hline ALL & 0.27 & 0.47 & 0.57 & 0.02 & 5.40 & 439 \\
\hline
\end{tabular}


Table $2 g$

Variation in Sodium Concentration (ppm) by $\mathrm{pH}$

$\mathrm{ph}$
$3.00-3.99$
$4.00-4.99$
$5.00-5.99$
$6.00-6.99$

wt

ALL

mean
0.54
0.76
0.47
1.51
0.64

\begin{tabular}{c} 
mean \\
\hline 1.09 \\
1.08 \\
0.66 \\
1.81
\end{tabular}

$\frac{\text { S. D. }}{2.96}$

$\frac{\min .}{0.06}$

$\frac{\max }{20.50}$

$\frac{\mathrm{n}}{47}$

1.82

0.01

15.40

220

0.96

0.02

5.75

118

4.54

0.09

24.80

30

2.13

0.01

24.80

415

\section{Table $2 \mathrm{~h}$}

Variation in Chloride Concentration (ppm) by $\mathrm{pH}$

$\frac{\mathrm{pH}}{3.00-3.99}$

wt. mean

\begin{tabular}{l} 
mean \\
\hline 2.48 \\
2.63 \\
1.99 \\
3.95 \\
6.70 \\
2.55
\end{tabular}

$\frac{\text { S. D. }}{7.02}$

min.

$\max$

1.21

5.07

0.20

49.50

$\frac{\mathrm{n}}{48}$

4.00-4.99

1.74

2.75

0.20

46.70

226

5.00-5.99

1.35

8.32

0.20

19.00

119

6.00-6.99

3.17

$-$

0.20

46.50

35

7.00-7.99

6.70

2.55

5.18

6.70

6.70

1

ALL

1.56

0.20

49.50

429

Table $2 i$

Variation in Nitrate/Sulfate Ratin by $\mathrm{pH}$

$\frac{\mathrm{pH}}{3.00-3.99}$ wt. mean
0.12
$\frac{\text { mean }}{0.19}$
0.10
0.15
0.03
0.08
0.06
0.10
0.08
0.13

$\frac{\text { S. D. }}{0.2 \theta}$

$\frac{\min .}{0.01}$

$\frac{\max }{1.05}$

$\frac{n}{48}$

$4.00-4.99$

0.12

0.00

0.72

224

$5.00-5.99$

0.07

0.01

0.38

119

$6.00-6.99$

0.07

0.02

0.29

32

ALL

0.12

0.00

1.05

423

Table $2 j$

Variation in Ammonium/Sulfate Ratio by $\mathrm{pH}$

$\frac{\mathrm{pH}}{3.00-3.99}$
$4.00-4.99$
$5.00-5.99$
$6.00-6.99$

ALL

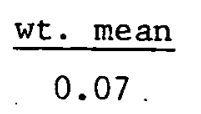

$\frac{\text { mean }}{0.11}$

0.08

0.10

0.04

0.14

0.07

0.10

0.22

0.11

\begin{tabular}{l} 
S. D. \\
\hline 0.15 \\
0.06 \\
0.11 \\
0.19
\end{tabular}

0.11

\begin{tabular}{l} 
min. \\
\hline 0.02 \\
0.02 \\
0.01 \\
0.04
\end{tabular}

0.01

\begin{tabular}{lr}
$\max$. & \multicolumn{1}{c}{ n } \\
1.07 & 47 \\
0.33 & 226 \\
0.81 & 122 \\
0.94 & 34 \\
1.07 & 429
\end{tabular}


Table 2k

Variation in Ammonium/Nitrate Ratio by $\mathrm{pH}$

\begin{tabular}{|c|c|c|c|c|c|}
\hline $\mathbf{p k}_{\mathbf{k}}$ & wt. mean & mean & S. D. & min. & max. \\
\hline $3.00-3.99$ & 0.80 & 0.98 & 1.53 & 0.21 & 8.64 \\
\hline $4.00-4.99$ & 1.13 & 1.14 & 2.12 & 0.09 & 21.00 \\
\hline $5.00-5.99$ & 1.80 & 1.68 & 2.02 & 0.28 & 20.00 \\
\hline $6.00-6.99$ & 2.64 & 2.63 & 1.70 & 0.47 & 8.50 \\
\hline ALL & 1.34 & 1.38 & 2.05 & 0.09 & 21.00 \\
\hline
\end{tabular}

Table 21

Variation in Sulfate/Sodium Ratio by $\mathrm{pH}$

$\frac{\mathrm{pH}}{3.00-3.99}$

4.00-4.99

5.00-5.99

6.00-6.99

ALL

$\mathrm{PH}$

3.00-3.99

4.00-4.99

5.00-5.99

6.00-6.99

ALL

$\begin{array}{rr}\text { wt. mean } & \text { mean } \\ 47.09 & 36.44 \\ 16.45 & 14.54 \\ 14.69 & 13.50 \\ 5.05 & 7.77 \\ 21.48 & 16.17\end{array}$

\begin{tabular}{r} 
S. D. \\
\hline 40.91 \\
21.05 \\
17.36 \\
7.20 \\
23.64
\end{tabular}

$\underline{\max }$

200.00

0.43

0.38

0.34

0.34

160.00

28.64

200.00 $\frac{\mathrm{n}}{45}$

45

215

117

30

407

\section{Table 2m}

Variation in Sulfate/Chloride Ratio by $\mathrm{pH}$

\begin{tabular}{|c|c|c|c|c|c|}
\hline wt. mean & mean & S. D. & min. & $\max$ & $n$ \\
\hline 12.56 & 10.27 & 6.98 & 0.28 & 32.91 & 47 \\
\hline 4.10 & 3.99 & 3.80 & 0.22 & 28.50 & 221 \\
\hline 3.79 & 3.12 & 2.35 & 0.11 & 10.00 & 118 \\
\hline 2.05 & 2.95 & 2.25 & 0.15 & 10.00 & 33 \\
\hline 5.58 & 4.37 & 4.41 & 0.11 & 32.91 & 419 \\
\hline
\end{tabular}

Table 2n.

Variation in Chloride/Sodium Ratio by $\mathrm{pH}$

\begin{tabular}{|c|c|c|c|c|c|c|}
\hline $\mathrm{pH}$ & wt. mean & xnean & S. D. & $\min$. & $\max$ & $\mathrm{n}$ \\
\hline $3.00-3.99$ & 3.58 & $\overline{3.43}$ & 3.00 & 1.21 & 18.18 & 46 \\
\hline $4.00-4.99$ & 3.74 & 3.46 & 4.12 & 0.19 & 50.00 & 217 \\
\hline $5.00-5.99$ & 4.02 & 4.05 & 3.88 & 1.29 & 30.00 & 113 \\
\hline $6.00-6.99$ & 2.30 & 2.50 & 1.22 & 1.28 & 7.73 & 30 \\
\hline ALL & 3.76 & 3.55 & 3.81 & 0.19 & 50.00 & 406 \\
\hline
\end{tabular}


Table 3a

Variation of $\mathrm{pH}$ by Season

\begin{tabular}{|c|c|c|c|c|c|}
\hline season & wt. mean & mean & min. & max. & $\underline{\mathrm{n}}$. \\
\hline winter & 4.66 & 4.50 & 3.74 & 7.20 & 124 \\
\hline spring & 4.37 & 4.36 & 3.64 & 6.66 & 151 \\
\hline summer & 4.0 .3 & 4.06 & 3.21 & 6.62 & 92 \\
\hline fall & 4.68 & 4.65 & 3.68 & 7.24 & 156 \\
\hline annual & 4.30 & 4.38 & 3.21 & 7.24 & 523 \\
\hline
\end{tabular}

$\underline{\text { Table 3b }}$

Variation of $\mathrm{pH}$ by Synoptic Type

\begin{tabular}{|c|c|c|c|c|c|}
\hline type & wt. mean & mean & min. & $\max$. & $\mathrm{n}$ \\
\hline low & 4.45 & 4.39 & 3.21 & 7.06 & 89 \\
\hline warm front & 4.58 & 4.48 & 3.32 & 7.24 & 298 \\
\hline cold front & 4.02 & 4.17 & 3.26 & 6.70 & 80 \\
\hline occluded front & 4.69 & 4.82 & 4.04 & 6.62 & 20 \\
\hline squall line & 3.88 & 4.01 & 3.48 & 6.52 & 27 \\
\hline hurricane & 5.54 & 5.57 & 5.36 & 5.96 & 8 \\
\hline al1 & 4.30 & 4.38 & 3.21 & 7.24 & 523 \\
\hline
\end{tabular}

$\underline{\text { Table } 3 \mathrm{c}}$

Variation of $\mathrm{pH}$ by Precipitation Type

\begin{tabular}{|c|c|c|c|c|c|}
\hline type & wt. mean & mean & min. & $\underline{\max }$. & $\mathrm{n}$ \\
\hline rain & 4.53 & 4.52 & 3.32 & 7.24 & 361 \\
\hline rain shower & 4.31 & 4.16 & 3.56 & 6.70 & 61 \\
\hline thundershower & 3.98 & 3.91 & 3.21 & 6.36 & 39 \\
\hline snow & 4.51 & 4.49 & 3.82 & 6.09 & 62 \\
\hline all & 4.30 & 4.38 & 3.21 & 7.24 & 523 \\
\hline
\end{tabular}

$\underline{\text { Table } 3 d}$

Variation of $\mathrm{pH}$ by Precipitation Rate

\begin{tabular}{|c|c|c|c|c|c|}
\hline rate (in./hour) & wt. mean & mean & min. & $\underline{\max }$. & $\mathrm{n}$ \\
\hline $0.00-0.03$ & 4.32 & 4.39 & 3.26 & 7.24 & 257 \\
\hline $0.04-0.10$ & 4.36 & 4.35 & 3.21 & 6.40 & 175 \\
\hline $0.11-0.25$ & 4.52 & 4.51 & 3.66 & 6.70 & 59 \\
\hline $0.26-0.50$ & 4.29 & 4.30 & 3.48 & 5.92 & 23 \\
\hline$\geq 0.51$ & 4.06 & 4.17 & 3.72 & 5.46 & 9 \\
\hline a11 & 4.30 & 4.38 & 3.21 & 7.24 & 523 \\
\hline
\end{tabular}


Table 3e

wind speed

Variation of $\mathrm{pH}$ by Wind Speed at $108 \mathrm{~m}$

\begin{tabular}{c}
$\left(\mathrm{m} \sec ^{-1}\right)$ \\
\hline $0-2$ \\
$3-5$ \\
$6-9$ \\
$\geq 10$
\end{tabular}

Wt. mean

mean

4.45

4.37

4.10

4.30

4.33

4.35

4.69

4.78

4.38

min.

3.63

3.21

3.26

3.98

3.21

max.

6.69

26

7.06

210

7.24

191

7.20

96

al1

4.30

7.24

523

Table $3 f$

Varlation of $\mathrm{pH}$ by Wind Direction

\begin{tabular}{|c|c|c|c|c|c|}
\hline direction & wt. mean & mean & min. & $\underline{\max }$. & $\mathrm{n}$ \\
\hline $\mathrm{N}$ & 4.78 & 4.75 & 4.25 & 6.12 & 42 \\
\hline $\mathrm{NE}$ & 4.59 & 4.49 & 3.68 & 6.66 & 69 \\
\hline $\mathrm{E}$ & 4.52 & 4.44 & 3.41 & 7.06 & 93 \\
\hline SE & 4.24 & 4.26 & 3.32 & 7.20 & 89 \\
\hline S & 4.52 & 4.41 & 3.56 & 6.99 & 71 \\
\hline SW & 4.13 & 4.16 & 3.21 & 7.24 & 70 \\
\hline W & 4.01 & 4.46 & 3.71 & 6.39 & 28 \\
\hline NW & 3.96 & 4.45 & 3.48 & 6.28 & 61 \\
\hline à11 & 4.30 & 4.38 & 3.21 & 7.24 & 523 \\
\hline
\end{tabular}

Table $3 g$

Variation of $\mathrm{pH}$ by Temperature

\begin{tabular}{l} 
temperature $\left({ }^{\circ} \mathrm{F}\right)$ \\
\hline $10-19$ \\
$20-29$ \\
$30-39$ \\
$40-49$ \\
$50-59$ \\
$60-69$ \\
$70-79$ \\
$80-89$ \\
al1
\end{tabular}

\begin{tabular}{ll} 
wt. mean & mean \\
\cline { 1 - 1 } 4.33 & 4.33 \\
4.46 & 4.47 \\
4.53 & 4.49 \\
4.52 & 4.46 \\
4.47 & 4.48 \\
4.38 & 4.41 \\
4.00 & 4.06 \\
3.98 & 3.98 \\
4.30 & 4.38
\end{tabular}

$\begin{array}{llr}\text { min. } & \text { max. } & \text { n } \\ 4.33 & 4.33 & 1 \\ 3.82 & 6.10 & 38 \\ 3.83 & 5.59 & 76 \\ 3.74 & 6.70 & 140 \\ 3.64 & 7.20 & 106 \\ 3.21 & 7.24 & 89 \\ 3.26 & 7.06 & 72 \\ 3.98 & 3.98 & 1 \\ 3.21 & 7.24 & 523\end{array}$ 
Table $4 a$

Variation in Hydrogen Ion Concentration $\times 10^{-4} \mathrm{M}$

by Season

\begin{tabular}{llllllr} 
seasun & wt. mean & mean & S. D. & min. & max. & n \\
\cline { 2 - 3 } & 0.22 & 0.32 & 0.37 & 0.00 & 1.82 & 124 \\
spring & 0.43 & 0.44 & 0.42 & 0.00 & 2.29 & 151 \\
summer & 0.93 & 0.87 & 1.37 & 0.00 & 6.17 & 92 \\
fal1 & 0.21 & 0.22 & 0.37 & 0.00 & 2.09 & 156 \\
annual & 0.51 & 0.42 & 0.71 & 0.00 & 6.17 & 523
\end{tabular}

Table $4 \mathrm{~b}$

Variation in Hydrogen Ion Concentration $\times 10^{-4} \mathrm{M}$ by Synoptic Type

\begin{tabular}{|c|c|c|c|c|c|c|}
\hline type & wt. mean & mean & $\underline{\text { S. D. }}$ & min. & $\underline{\max }$. & $\mathrm{n}$ \\
\hline low & 0.36 & 0.41 & 0.85 & 0.00 & 6.17 & 89 \\
\hline warm front & 0.27 & 0.33 & 0.49 & 0.00 & 4.79 & 298 \\
\hline cold front & 0.96 & 0.67 & 1.01 & 0.00 & 5.50 & 80 \\
\hline occluded front & 0.20 & 0.15 & 0.23 & 0.00 & 0.91 & 20 \\
\hline squall line & 1.33 & 0.98 & 0.97 & 0.00 & 3.31 & 27 \\
\hline hurricane & 0.03 & 0.03 & 0.01 & 0.01 & 0.04 & 8 \\
\hline all & 0.51 & 0.42 & 0.71 & 0.00 & 6.17 & 523 \\
\hline
\end{tabular}

Table $4 c$

Variation in Hydrogen Ion Concentration $\times 10^{-4} \mathrm{M}$

by Precipitation Type

\begin{tabular}{|c|c|c|c|c|c|c|}
\hline type & wt. mean & mean & S. D. & min. & $\max$. & $\mathbf{n}$ \\
\hline rain & 0.30 & 0.30 & 0.50 & 0.00 & 4.79 & 361 \\
\hline rain shower & 0.49 & 0.70 & 0.78 & 0.00 & 2.75 & 61 \\
\hline thundershower & 1.05 & 1.22 & 1.59 & 0.00 & 6.17 & 39 \\
\hline snow & 0.31 & 0.32 & 0.32 & 0.01 & 1.51 & 62 \\
\hline al1 & 0.51 & 0.42 & 0.71 & 0.00 & 6.17 & 523 \\
\hline
\end{tabular}

Table 4d

Variation in Hydrogen Ion Concentration $\times 10^{-4} \mathrm{M}$

by Precipitation Rate

\begin{tabular}{|c|c|c|c|c|c|c|}
\hline rate (in./hour) & wt. mean & mean & S. D. & min. & $\max$. & $\mathbf{n}$ \\
\hline $0.00-0.03$ & 0.47 & 0.41 & 0.80 & 0.00 & 5.50 & 257 \\
\hline $0.04-0.10$ & 0.43 & 0.45 & 0.63 & 0.00 & 6.17 & 175 \\
\hline $0.11-0.25$ & 0.31 & 0.31 & 0.38 & 0.00 & 2.19 & 59 \\
\hline $0.26-0.50$ & 0.51 & 0.51 & 0.81 & 0.01 & 3.31 & 23 \\
\hline$\therefore 0.51$ & 0.88 & 0.68 & 0.73 & 0.04 & 1.91 & 9 \\
\hline a11 & 0.51 & 0.42 & 0.71 & 0.00 & 6.17 & 523 \\
\hline
\end{tabular}


Table $4 \mathrm{e}$

Variation in Hydrogen Ion Concentration $\times 10^{-4} \mathrm{M}$

by wind Speed at $108 \mathrm{~m}$

\begin{tabular}{l} 
wind speed \\
(m sec-1) \\
\hline $0-2$ \\
$3-5$ \\
$6-9$ \\
210 \\
all
\end{tabular}

\begin{tabular}{lllllr} 
wt. mean & mean & S. D. & min. & max. & n \\
\cline { 1 - 2 } 0.36 & 0.42 & 0.58 & 0.00 & 2.34 & 26 \\
0.80 & 0.50 & 0.86 & 0.00 & 6.17 & 210 \\
0.47 & 0.45 & 0.68 & 0.00 & 5.50 & 191 \\
0.17 & 0.20 & 0.23 & 0.00 & 1.05 & 96 \\
0.51 & 0.42 & 0.71 & 0.00 & 6.17 & 523
\end{tabular}

Table $4 \mathrm{f}$

Variation in Hydrogen Ion Concentration $\times 10^{-4} \mathrm{M}$ by Wind Direction

\begin{tabular}{|c|c|c|c|c|c|c|}
\hline direction & wt. mean & mean & S. D. & min. & $\underline{\max }$. & $\mathrm{n}$ \\
\hline $\mathrm{N}$ & 0.17 & 0.18 & 0.19 & 0.01 & 1.00 & 42 \\
\hline $\mathrm{NE}$ & 0.26 & 0.32 & 0.40 & 0.00 & 2.09 & 69 \\
\hline $\mathrm{E}$ & 0.30 & 0.36 & 0.50 & 0.00 & 3.89 & 93 \\
\hline$\overline{S E}$ & 0.57 & 0.56 & 0.86 & 0.00 & 4.79 & 89 \\
\hline$S$ & 0.30 & 0.39 & 0.57 & 0.00 & 2.75 & 71 \\
\hline SW & 0.75 & 0.69 & 1.21 & 0.00 & 6.17 & 70 \\
\hline W & 0.97 & 0.34 & 0.55 & 0.00 & 1.95 & 28 \\
\hline NW & 1.11 & 0.36 & 0.58 & 0.01 & 3.31 & 61 \\
\hline all & 0.51 & 0.42 & 0.71 & 0.00 & 6.17 & 523 \\
\hline
\end{tabular}

Table $4 \mathrm{~g}$

Variation in Hydrogen Ion Concentration $\times 10^{-4} \mathrm{M}$

by Temperature

\begin{tabular}{|c|c|c|c|c|c|c|}
\hline temperature $\left({ }^{\circ} \mathrm{F}\right)$ & wt. mean & mean & S. D. & min. & $\underline{\max }$. & n. \\
\hline $10-19$ & 0.47 & 0.47 & - & 0.47 & 0.47 & 1 \\
\hline $20-29$ & 0.35 & 0.34 & 0.34 & 0.01 & 1.51 & 38 \\
\hline $30-39$ & 0.30 & 0.32 & 0.30 & 0.03 & 1.48 & 76 \\
\hline $40-49$ & 0.30 & 0.35 & 0.36 & 0.00 & 1.82 & 140 \\
\hline $50-59$ & 0.34 & 0.33 & 0.47 & 0.00 & 2.29 & 106 \\
\hline $60-69$ & 0.42 & 0.39 & 0.80 & 0.00 & 6.17 & 89 \\
\hline $70-79$ & 0.99 & 0.87 & 1.38 & 0.00 & 5.50 & 72 \\
\hline $80-89$ & 1.05 & 1.05 & - & 1.05 & 1.05 & 1 \\
\hline all & 0.51 & 0.42 & 0.71 & 0.00 & 6.17 & 523 \\
\hline
\end{tabular}




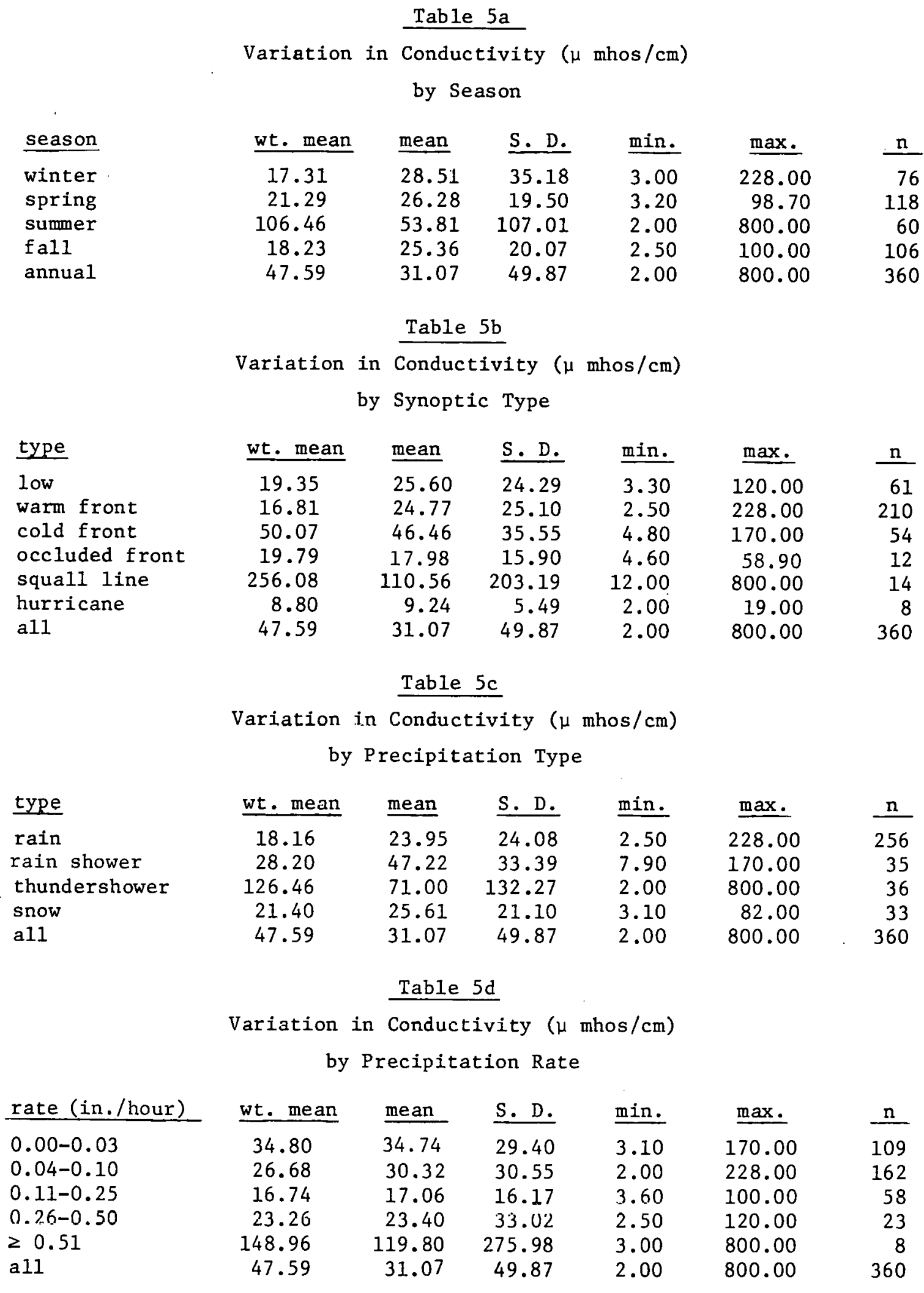


Table 5e

Variation in Conductivity ( $\mu \mathrm{mhos} / \mathrm{cm}$ )

by Wind speed at $108 \mathrm{~m}$

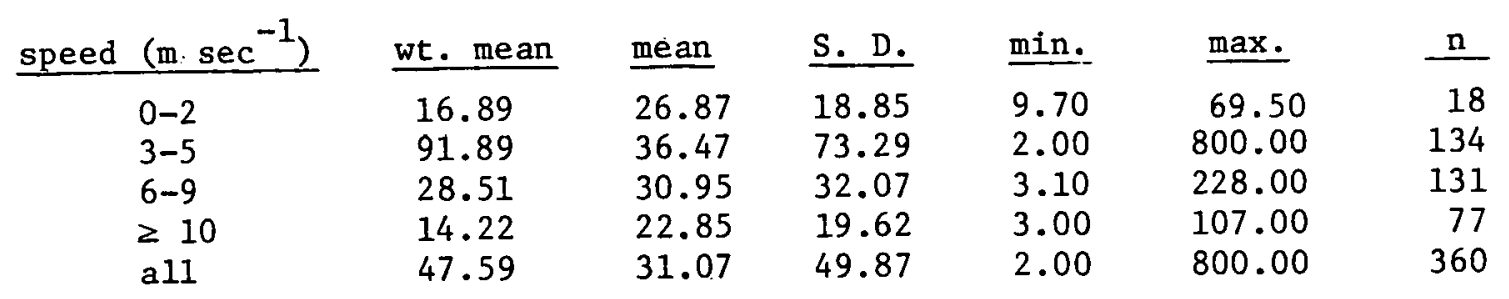

Table $5 f$

Variation in Conductivity ( $\mu \operatorname{mhos} / \mathrm{cm}$ ) by Wind Direction

\begin{tabular}{crrrrrr} 
direction & wt. mean & mean & S. D. & min. & max. & n \\
\cline { 2 - 3 } N & 19.02 & 21.66 & 13.23 & 4.70 & 54.00 & 29 \\
NE & 15.25 & 23.52 & 21.49 & 2.00 & 100.00 & 47 \\
E & 15.80 & 22.95 & 18.89 & 3.10 & 100.00 & 68 \\
SE & 31.26 & 35.42 & 39.55 & 2.50 & 228.00 & 64 \\
S & 19.81 & 28.37 & 29.30 & 4.80 & 170.00 & 54 \\
SW & 39.38 & 44.32 & 34.38 & 3.30 & 170.00 & 43 \\
W & 49.47 & 31.41 & 31.79 & 7.60 & 120.00 & 15 \\
NW & 246.90 & 42.86 & 125.12 & 3.10 & 800.00 & 40 \\
a11 & 47.59 & 31.07 & 49.87 & 2.00 & 800.00 & 360
\end{tabular}

Table 5g

Variation in Conductivity ( $\mu$ mhos $/ \mathrm{cm}$ )

by Temperature

\begin{tabular}{|c|c|c|c|c|c|c|}
\hline temperature $\left({ }^{\circ} \mathrm{F}\right)$ & wt. mean & mean & S. D. & min. & $\max$. & $\underline{n}$ \\
\hline $20-29$ & 24.91 & 27.41 & 20.45 & 8.00 & 82.00 & 18 \\
\hline $30-39$ & 20.58 & 26.24 & 18.34 & 3.10 & 90.00 & 54 \\
\hline $40-49$ & 15.07 & 23.08 & 26.14 & 3.20 & 228.00 & 102 \\
\hline $50-59$ & 23.13 & 30.43 & 27.55 & 3.00 & 163.00 & 77 \\
\hline $60-69$ & 22.86 & 30.55 & 28.37 & 2.00 & 120.00 & 61 \\
\hline $70-79$ & 128.21 & 56.66 & 119.22 & 4.10 & 800.00 & 47 \\
\hline $80-89$ & 49.40 & 49.40 & - & 49.40 & 49.40 & 1 \\
\hline all & 47.59 & 31.07 & 49.87 & 2.00 & 800.00 & 360 \\
\hline
\end{tabular}


Table 6a

Variation in Excess Conductivity ( $\mu$ mhos $/ \mathrm{cm}$ )

by Season

\begin{tabular}{|c|c|c|c|c|c|c|}
\hline season & wt. mean & mean & S. D. & $\min$. & max. & n \\
\hline winter & 10.79 & 18.84 & 28.54 & 1.09 & 183.90 & 76 \\
\hline spring & 7.26 & 10.96 & 12.89 & 0.00 & 54.74 & 118 \\
\hline summer & 75.63 & 27.60 & 94.55 & 0.00 & 733.15 & 60 \\
\hline fall & 10.85 & 15.60 & 10.55 & 2.07 & 55.38 & 106 \\
\hline annual & 30.88 & 16.76 & 41.93 & 0.00 & 733.15 & 360 \\
\hline
\end{tabular}

Table 6b

Variation in Excess Conductivity ( $\mu \mathrm{mhos} / \mathrm{cm}$ )

by Synoptic Type

\begin{tabular}{|c|c|c|c|c|c|c|}
\hline type & wt. mean & mean & S. D. & min. & max. & $\mathrm{n}$ \\
\hline low & 9.87 & 12.18 & 9.60 & 0.00 & 49.98 & 61 \\
\hline warm front & 8.59 & 14.07 & 20.28 & 0.00 & 183.90 & 210 \\
\hline cold front & 16.52 & 20.71 & 17.00 & 0.00 & 78.67 & 54 \\
\hline occluded front & 11.24 & 10.97 & 8.62 & 0.00 & 27.48 & 12 \\
\hline squall line & 209.06 & 72.06 & 191.33 & 0.00 & 733.15 & 14 \\
\hline hurricane & 7.79 & 8.30 & 5.39 & 1.08 & 17.70 & 8 \\
\hline all & 30.88 & 16.76 & 41.93 & 0.00 & 733.15 & 360 \\
\hline
\end{tabular}

Table 6c

Variation in Excess Conductivity ( $\mu \mathrm{mhos} / \mathrm{cm}$ )

by Precipitation Type

\begin{tabular}{|c|c|c|c|c|c|c|}
\hline type & wt. mean & mean & S. D. & $\underline{\min }$ : & $\underline{\max }$. & $\mathrm{n}$ \\
\hline rain & 8.85 & 13.59 & 17.94 & 0.00 & 183.90 & 256 \\
\hline rain shower & 11.90 & 21.98 & 16.22 & 0.00 & 73.75 & 35 \\
\hline thundershower & 91.09 & 36.01 & 121.43 & 0.00 & 733.15 & 36 \\
\hline snow & 11.67 & 14.86 & 14.85 & 0.00 & 64.86 & 33 \\
\hline al! & 30.88 & 16.76 & 41.93 & 0.00 & 733.15 & 360 \\
\hline
\end{tabular}

Table 6d

Variation in Excess Conductivity ( $\mu \mathrm{mhos} / \mathrm{cm}$ )

by Precipitation Rate

\begin{tabular}{lcrrrrr} 
rate (in./hour) & wt. mean & mean & S. D. & min. & max. & n \\
\hline $0.00-0.03$ & 17.57 & 19.00 & 17.51 & 0.00 & 136.44 & 109 \\
$0.04-0.10$ & 15.27 & 16.28 & 20.96 & 0.00 & 183.90 & 162 \\
$0.11-0.25$ & 6.73 & 6.81 & 7.01 & 0.00 & 25.44 & 58 \\
$0.26-0.50$ & 5.98 & 6.32 & 8.50 & 0.00 & 30.65 & 23 \\
20.51 & 119.31 & 98.33 & 256.59 & 1.32 & 733.15 & 8 \\
al1 & 30.88 & 16.76 & 41.93 & 0.00 & 733.15 & 360
\end{tabular}


Table 6e

Variation in Excess Conductivity $(\mu$ mhos $/ \mathrm{cm}$ )

by Wind Speed at $108 \mathrm{~m}$

wind speed

$(\mathrm{m} \mathrm{sec}-1)$

$0-2$

3-5

6-9

$\geq 10$

a11 wt. mean

5.01

64.74

12.52

8.80

30.88

mean
11.03
18.91
16.19
15.35
16.76

16.76
S. D.

9.12

63.52

23.43

16.39

41.93

min.
0.00
0.00
0.00
0.00
0.00

max.

32.74

733.15

183.90

91.71

733.15



360

\section{Table $6 f$}

Variation in Excess Conductivity $(\mu$ mhos $/ \mathrm{cm}$ )

by Wind Direction

\begin{tabular}{|c|c|c|c|c|c|c|}
\hline direction & wt. mean & mean & S. D. & min. & $\underline{\max }$. & $\mathrm{n}$ \\
\hline $\mathrm{N}$ & 13.15 & 14.68 & 9.06 & 4.08 & 49.98 & 29 \\
\hline $\mathrm{NE}$ & 6.79 & 10.55 & 11.19 & 0.00 & 41.40 & 47 \\
\hline $\mathrm{E}$ & 6.30 & 10.40 & 11.33 & 0.00 & 64.86 & 68 \\
\hline $\mathrm{SE}$ & 12.39 & 19.81 & 30.58 & 0.00 & 183.90 & 64 \\
\hline $\mathrm{S}$ & 10.24 & 15.57 & 17.13 & 0.00 & 78.67 & 54 \\
\hline SW & 15.73 & 20.66 & 18.05 & 0.00 & 82.85 & 43 \\
\hline W & 17.67 & 16.04 & 14.17 & 0.00 & 51.75 & 15 \\
\hline NW & 206.11 & 29.22 & 114.50 & 0.00 & 733.15 & 40 \\
\hline a11 & 30.88 & 16.76 & 41.93 & 0.00 & 733.15 & 360 \\
\hline
\end{tabular}

\section{Table $6 \mathrm{~g}$}

Variation in Excess Conductivity ( $\mu \mathrm{mhos} / \mathrm{cm}$ )

by Temperature

\begin{tabular}{|c|c|c|c|c|c|c|}
\hline temperature $\left({ }^{\circ} \mathrm{F}\right)$ & wt. mean & mean & S. D. & min. & $\underline{\max }$. & $\mathrm{n}$ \\
\hline $20-29$ & 13.15 & 14.56 & 10.55 & 3.58 & 37.90 & 18 \\
\hline $30-39$ & 10.78 & 15.41 & 14.57 & 0.00 & 64.86 & 54 \\
\hline $40-49$ & 5.64 & 11.11 & 20.44 & 0.00 & 183.90 & 102 \\
\hline $50-59$ & 11.41 & 16.94 & 20.02 & 0.00 & 136.44 & 77 \\
\hline $60-69$ & 11.35 & 16.12 & 14.70 & 0.00 & 82.85 & 61 \\
\hline $70-79$ & 94.40 & 32.08 & 106.11 & 0.00 & 733.15 & 47 \\
\hline $80-89$ & 12.65 & 12.65 & - & 12.65 & 12.65 & 1 \\
\hline al1 & 30.88 & 16.76 & 41.93 & 0.00 & 733.15 & 360 \\
\hline
\end{tabular}


Table 7a

Variation in Sulfate Concentration (ppm)

by Season

season

winter

spring

summer

fall

annual wt. mean

\subsection{4}

2.97

5.40

2.82

3.72

$\begin{array}{lr}\text { mean } & \text { S. D. } \\ 3.85 & 2.80 \\ 3.85 & 3.16 \\ 8.09 & 16.97 \\ 3.81 & 2.62 \\ 4.55 & 7.53\end{array}$

min.
2.00
2.00
2.49
1.11
1.11

$\underline{\max }$

14.67

26.30

142.50

16.29

142.50

II
135
73
133
439

Table 7b

Variation in Sulfate Concentration (ppm)

by Synoptic Type

type
low
warm front
cold front
occluded front
squall line
hurricane
all

\begin{tabular}{c} 
wt. mean \\
\hline 3.00 \\
2.72 \\
5.99 \\
2.49 \\
6.43 \\
2.50 \\
3.72
\end{tabular}

\begin{tabular}{l} 
mean \\
\hline 3.59 \\
3.74 \\
8.16 \\
2.43 \\
8.54 \\
2.52 \\
4.55
\end{tabular}

S. D.

2.93

min.

1. 11

2. 84

2.00

17.24

2. 00

0.58

2.00

6.35

2.49

0.07

2.49

7.53

1.11

$\underline{\max }$.

15.15

27.00

142.50

4.30

23.04

2.70

142.50

\begin{tabular}{r}
\multicolumn{1}{c}{ n } \\
77 \\
251 \\
68 \\
15 \\
19 \\
8 \\
439
\end{tabular}

Table 7c

Variation in Sulfate Concentration (ppm)

by Precipitation Type

type
rain
rain shower
thundershower
snow

a11

\begin{tabular}{|c|c|}
\hline mean & S. D. \\
\hline 3.61 & 2.85 \\
\hline 10.39 & 20.50 \\
\hline 6.02 & 4.76 \\
\hline 3.67 & 2.18 \\
\hline 4.55 & 7.53 \\
\hline
\end{tabular}

Table $7 d$

Variation in Sulfate Concentration (ppm)

by Precipitation Rate

\begin{tabular}{|c|c|c|c|c|c|c|}
\hline rate (in./hour) & wt. mean & mean & S. D. & min. & $\underline{\max }$. & $\mathrm{n}$ \\
\hline $0.00-0.03$ & 5.61 & 6.14 & 11.12 & 1.11 & 142.50 & 179 \\
\hline $0.04-0.10$ & 3.52 & 3.71 & 3.19 & 1.29 & 22.50 & 169 \\
\hline $0.11-0.25$ & 2.60 & 2.62 & 1.17 & 2.00 & 7.95 & 59 \\
\hline $0.26-0.50$ & 3.45 & 3.46 & 2.82 & 2.00 & 12.96 & 23 \\
\hline$\geq 0.51$ & 4.93 & 3.98 & 2.84 & 2.00 & 9.90 & 9 \\
\hline a 11 & 3.72 & 4.55 & 7.53 & 1.11 & 142.50 & 439 \\
\hline
\end{tabular}


Table 7e

Variation in Sulfate Concentration (ppm)

by Wind Speed at $108 \mathrm{~m}$

wind speed

$(\mathrm{m} \mathrm{sec}-1)$

$0-2$

$3-5$

6-9

$\geq 10$

a11

\begin{tabular}{c} 
wt. mean \\
\hline 2.59 \\
4.83 \\
3.89 \\
2.30 \\
3.72
\end{tabular}

\begin{tabular}{l} 
mean \\
\hline 3.54 \\
4.89 \\
5.33 \\
2.66 \\
4.55
\end{tabular}

S. D.

\begin{tabular}{l} 
min. \\
\hline 2.00 \\
1.29 \\
1.11 \\
2.00 \\
1.11
\end{tabular}

$\max$.

7.75

$\underline{\mathrm{n}}$

27.00

21

163

4.31

142.50

167

7.90

142.50

88

439

Table $7 f$

Variation in Sulfate Concentration (ppm)

by Wind Direction

direction

$\mathrm{N}$

$\mathrm{NE}$

$\mathrm{E}$

SE

$S$

SW

W

NW

al1

\begin{tabular}{c} 
wt. mean \\
\hline 2.57 \\
2.85 \\
2.71 \\
3.91 \\
3.10 \\
5.38 \\
4.65 \\
6.11 \\
3.72
\end{tabular}

\begin{tabular}{l} 
mean \\
\hline 2.86 \\
3.62 \\
3.63 \\
4.81 \\
4.10 \\
8.86 \\
4.32 \\
3.76 \\
4.55
\end{tabular}

S. D.

min.

$\underline{\max }$.

n

35

57

79

76

63

55

24

50

439

\section{Table 7g}

Variation in Sulfate Concentration (ppm)

by Temperature

\begin{tabular}{l} 
temperature $\left({ }^{\circ} \mathrm{F}\right)$ \\
\hline $20-29$ \\
$30-39$ \\
$40-49$ \\
$50-59$ \\
$60-69$ \\
$70-79$ \\
$80-89$ \\
a11
\end{tabular}

\begin{tabular}{c} 
wt. mean \\
\hline 3.66 \\
2.98 \\
2.59 \\
2.98 \\
3.50 \\
5.76 \\
5.26 \\
3.72
\end{tabular}

$\frac{\text { mean }}{3.87}$
3.61
3.59
3.85
6.48
6.41
5.26
4.55

\begin{tabular}{c} 
S. D. \\
\hline 2.55 \\
2.06 \\
2.64 \\
2.60 \\
16.30 \\
6.01 \\
- \\
7.53
\end{tabular}

$\frac{\text { min }}{2.00}$
2.00
2.00
1.10
2.01
2.01
5.26
1.11

$\max$
13.30
14.67
14.20
12.80
142.50
27.00
5.26
142.50

\begin{tabular}{r}
\multicolumn{1}{c}{$\mathrm{n}$} \\
\hline 26 \\
66 \\
121 \\
90 \\
77 \\
58 \\
1 \\
439
\end{tabular}


Table 8a

Variation in Excess Sulfate Concentration (ppm)

by Season

\begin{tabular}{|c|c|c|c|c|c|c|}
\hline season & wt. mean & mean & S. D. & min. & $\max$. & $\mathrm{n}$ \\
\hline winter & 2.58 & 3.43 & 2.47 & 1.34 & 14.28 & 85 \\
\hline spring & 2.70 & 3.20 & 2.23 & 0.84 & 13.74 & 124 \\
\hline summer & 5.19 & 5.86 & 5.73 & 1.96 & 26.96 & 70 \\
\hline fall & 2.65 & 3.58 & 2.54 & 0.50 & 16.26 & 128 \\
\hline annual & 3.50 & 3.82 & 3.36 & 0.50 & 26.96 & 407 \\
\hline
\end{tabular}

Table $8 \mathrm{~b}$

Variation in Excess Sulfate Concentration (ppm)

by Synoptic Type

\begin{tabular}{lcccccr} 
type & wt. mean & mean & S. D. & min. & max. & n \\
\cline { 2 - 6 } low & 2.77 & 3.33 & 2.99 & 0.96 & 14.96 & 73 \\
warm front & 2.50 & 3.30 & 2.52 & 0.50 & 26.96 & 232 \\
cold front & 5.70 & 5.90 & 4.39 & 1.90 & 20.87 & 61 \\
occluded front & 2.17 & 2.09 & 0.62 & 0.84 & 3.50 & 15 \\
squall line & 6.31 & 7.72 & 6.26 & 2.40 & 23.01 & 17 \\
hurricane & 2.31 & 2.31 & 0.21 & 1.96 & .2 .56 & 8 \\
all & 3.50 & 3.82 & 3.36 & 0.50 & 26.96 & 407
\end{tabular}

Table 8c

Variation in Excess Sulfate Concentration (ppm)

by Precipitation Type

\begin{tabular}{|c|c|c|c|c|c|c|}
\hline type & wt. mean & mean & S. D. & $\underline{\min }$. & $\max$. & $\mathrm{n}$ \\
\hline rain & 2.58 & 3.19 & 2.59 & 0.50 & 26.96 & 291 \\
\hline rain shower & 4.19 & 6.80 & 4.96 & 1.84 & 23.01 & 41 \\
\hline thundershower & 5.49 & 5.97 & 4.78 & 1.98 & 17.74 & 36 \\
\hline snow & 3.10 & 3.42 & 2.23 & 1.73 & 13.02 & 39 \\
\hline a11 & 3.50 & 3.82 & 3.36 & 0.50 & 26.96 & 407 \\
\hline
\end{tabular}

Table 8d

Variation in Excess Sulfate Concentration (ppm)

by Precipitation Rate

\begin{tabular}{|c|c|c|c|c|c|c|}
\hline rate (in./hour) & wt. mean & mean & S. D. & min. & $\max$. & $\mathrm{n}$ \\
\hline $0.00-0.03$ & 4.80 & 4.87 & 3.98 & 0.96 & 26.96 & 150 \\
\hline $0.04-0.10$ & 3.24 & 3.42 & 3.12 & 0.50 & 21.90 & 166 \\
\hline $0.11-0.25$ & 2.46 & 2.47 & 1.16 & 1.42 & 7.88 & 59 \\
\hline $0.26-0.50$ & 3.34 & 3.35 & 2.76 & 1.62 & 12.82 & 23 \\
\hline 20.51 & 4.88 & 3.93 & 2.86 & 1.84 & 9.85 & 9 \\
\hline a11 & 3.50 & 3.82 & 3.36 & 0.50 & 26.96 & 407 \\
\hline
\end{tabular}


Table $8 \mathrm{e}$

Variation in Excess Sulfate Concentration (ppm)

by Wind Speed at $108 \mathrm{~m}$

wind speed

\begin{tabular}{l}
$(\mathrm{m} \mathrm{sec}-1)$ \\
\hline $0-2$ \\
$3-5$ \\
$6-9$ \\
210 \\
al1
\end{tabular}

\begin{tabular}{l} 
wt. mean \\
\hline 2.46 \\
4.77 \\
3.48 \\
2.03 \\
3.50
\end{tabular}

$\begin{array}{ll}\text { mean } & \text { S. D. } \\ 3.28 & 1.93 \\ 4.72 & 4.29 \\ 3.90 & 2.90 \\ 2.17 & 1.06 \\ 3.82 & 3.36\end{array}$

$\frac{\min .}{1.88}$

max.

$\underline{\mathrm{n}}$

1.27

7.61

19

152

153

0.96

26.96

20.87

0.50

7.73

83

407

Table $8 \mathrm{f}$

Variation in Excess Sulfate Concentration (ppm)

by Wind Direction

direction

$\mathrm{N}$

NE

E

$S E$

$S$

SW

W

NW

all

\begin{tabular}{ll} 
mean & S. D. \\
\hline 2.53 & 1.05 \\
3.41 & 2.56 \\
3.35 & 2.04 \\
4.16 & 4.31 \\
3.77 & 3.86 \\
5.56 & 3.83 \\
4.38 & 3.67 \\
3.48 & 3.26 \\
3.82 & 3.36
\end{tabular}

3.82 min.

0.96

1.27

1.45

1.34

0.50

1.84

1.73

0.84

0.50

26.96

Table $8 \mathrm{~g}$

Variation in Excess Sulfate Concentration (ppm)

by Temperature

\begin{tabular}{|c|c|c|c|c|c|c|}
\hline temperature $\left({ }^{\circ} \mathrm{F}\right)$ & wt. mean & mean & S. D. & min. & $\max$. & $\mathrm{n}$ \\
\hline $20-29$ & 3.57 & 3.75 & 2.66 & 1.88 & 13.02 & 22 \\
\hline $30-39$ & 2.69 & 3.22 & 2.09 & 0.84 & 14.28 & 61 \\
\hline $40-49$ & 2.38 & 3.03 & 1.97 & 1.45 & 13.74 & 108 \\
\hline $50-59$ & 2.74 & 3.59 & 2.50 & 0.96 & 12.72 & 86 \\
\hline $60-69$ & 3.11 & 4.04 & 3.49 & 0.50 & 20.87 & 72 \\
\hline $70-79$ & 5.65 & 6.05 & 5.93 & 1.09 & 26.96 & 57 \\
\hline $80-89$ & 5.24 & 5.24 & - & 5.24 & 5.24 & 1 \\
\hline all & 3.50 & 3.82 & 3.36 & 0.50 & 26.96 & 407 \\
\hline
\end{tabular}


Table $9 a$

Variation in Nitrate Concentration (ppm)

by Season

season
winter
spring
summer
fall
annual

type

low

warm front

cold front

occluded front

squall line

hurricane

al1

\begin{tabular}{c} 
wt. mean \\
\hline 0.38 \\
0.31 \\
0.43 \\
0.26 \\
0.36
\end{tabular}

\begin{tabular}{|c|c|c|}
\hline mean & S. D. & min. \\
\hline 0.81 & 0.91 & 0.02 \\
\hline 0.53 & 0.67 & 0.02 \\
\hline 0.62 & 0.83 & 0.02 \\
\hline 0.47 & 0.47 & 0.02 \\
\hline 0.59 & 0.72 & 0.02 \\
\hline
\end{tabular}

Table $9 \mathrm{~b}$

Variation in Nitrate Concentration (ppm) by Synoptic Type

\begin{tabular}{c} 
wt. mean \\
\hline 0.41 \\
0.24 \\
0.64 \\
0.36 \\
0.53 \\
0.06 \\
0.36
\end{tabular}

\begin{tabular}{l} 
mean \\
\hline 0.60 \\
0.52 \\
0.85 \\
0.32 \\
0.90 \\
0.06 \\
0.59
\end{tabular}

S. D.

0.89

min.

0.02

0.67

0.02

0.71

0.05

0.45

0.57

0.04

0.10

0.03

0.02

0.72

0.02

Table $9 \mathrm{c}$

Variation in Nitrate Concentration (ppm)

by Precipitation Type

type
rain
rain shower
thundershower
snow
al1

\begin{tabular}{lllll} 
wt. mean & mean & S. D. & min. \\
\cline { 1 - 1 } 0.23 & & 0.42 & 0.55 & 0.02 \\
0.48 & & 1.03 & 0.91 & 0.03 \\
0.51 & 0.80 & 0.85 & 0.02 \\
0.84 & 1.07 & 0.92 & 0.05 \\
0.36 & 0.59 & 0.72 & 0.02 \\
& & & \\
& & & \\
& & & \\
& & &
\end{tabular}

Variation in Nitrate Concentration (ppm)

by Precipitation Rate rate (in./hour)

$0.00-0.03$

0.04-0.10

$0.11-0.25$

$0.26-0.50$

$\geq 0.51$

all

\begin{tabular}{c} 
wt. mean \\
\hline 0.85 \\
0.48 \\
0.18 \\
0.24 \\
0.34 \\
0.36
\end{tabular}

\begin{tabular}{l} 
mean \\
\hline 0.86 \\
0.52 \\
0.19 \\
0.24 \\
0.26 \\
0.59
\end{tabular}

S. D.

0.82

min.

0.02

0.02

0.21

0.02

0.38

0.02

0.29

0.02

0.72

0.02 $\underline{\max }$.

4.30

3.53

4.10

2.58

4.30

\begin{tabular}{r}
\multicolumn{1}{c}{$n$} \\
97 \\
136 \\
72 \\
129 \\
434
\end{tabular}

36

72

129 
Table 9e

Variation in Nitrate Concentration (ppm)

wind speed

by Wind Speed at $108 \mathrm{~m}$

$\left(\mathrm{m} \mathrm{sec}^{-1}\right)$

$0-2$

3-5

$6-9$

210

al1

\begin{tabular}{l} 
wt. mean \\
\hline 0.31 \\
0.47 \\
0.46 \\
0.12 \\
0.36
\end{tabular}

\begin{tabular}{cc} 
mean & S. D. \\
\hline 0.83 & 0.97 \\
0.66 & 0.71 \\
0.66 & 0.75 \\
0.26 & 0.48 \\
0.59 & 0.72
\end{tabular}

0.02

max.

n

3.45

$4.10 \quad 163$

$4.30 \quad 165$

0.12

0.02

87

434

Table $9 \mathrm{f}$

Variation in Nitrate Concentration (ppm)

by Wind Direction

direction

$\mathrm{N}$

$\mathrm{NE}$

E

SE

$S$

SW

W

NW

al1 wt. mean

0.38

0.29

0.18

0.30

0.29

0.78

0.67

0.62

0.36

$\begin{array}{cc}\frac{\text { mean }}{0.58} & \text { S.D. } \\ 0.53 & 0.53 \\ 0.41 & 0.61 \\ 0.55 & 0.64 \\ 0.54 & 0.76 \\ 1.04 & 0.81 \\ 0.73 & 0.79 \\ 0.55 & 0.69 \\ 0.59 & 0.67\end{array}$

min.

0.02

0.02

0.02

0.02

0.03

0.03

0.19

0.05

0.02

3.00

4.30

\section{Table $9 \mathrm{~g}$ \\ Variation in Nitrate Concentration (ppm)}

by Temperature

\begin{tabular}{l} 
temperature $\left({ }^{\circ} \mathrm{F}\right)$ \\
\hline $20-29$ \\
$30-39$ \\
$40-49$ \\
$50-59$ \\
$60-69$ \\
$70-79$ \\
$80-89$ \\
a11
\end{tabular}

\begin{tabular}{c} 
wt. mean \\
\hline 1.03 \\
0.38 \\
0.25 \\
0.27 \\
0.29 \\
0.44 \\
0.98 \\
0.36
\end{tabular}

\begin{tabular}{c} 
mean \\
\hline 1.24 \\
0.60 \\
0.55 \\
0.45 \\
0.47 \\
0.68 \\
0.98 \\
0.59
\end{tabular}

S. D.

min.

0.91

0.17

max.

max.

2.43

2.75

4.30

4.10

3.60

3.28

3.00

3.00

4.30

\begin{tabular}{l} 
n \\
\hline 36 \\
57 \\
81 \\
74 \\
62 \\
51 \\
22 \\
51 \\
434
\end{tabular}
n
36
57
81
74
62
51
22
51
34 
Table 10a

Variation in Ammonium Concentration (ppm)

by Season

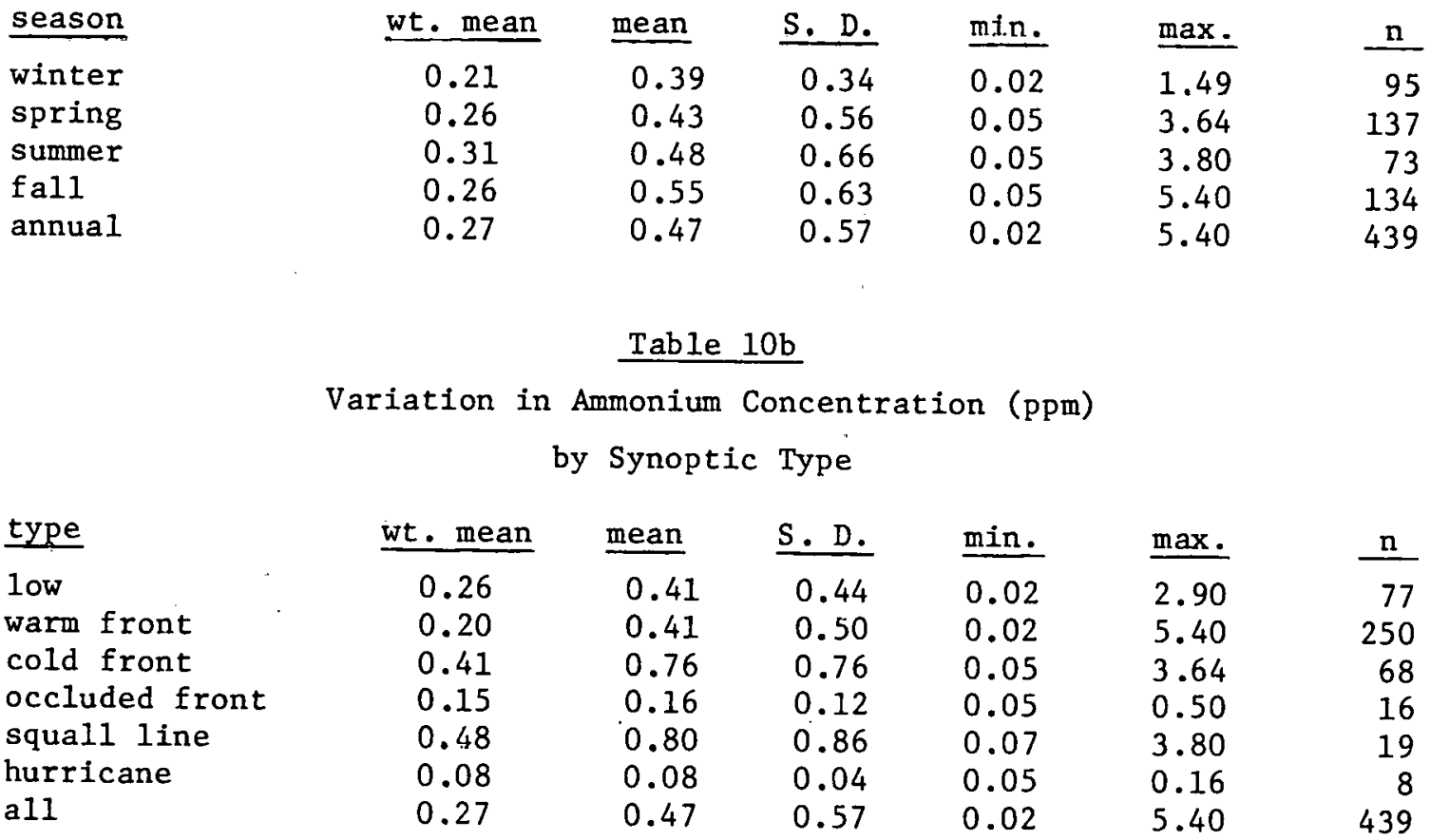

Table $10 c$

Variation in Ammonium Concentration (ppm)

by Precipitation Type

\begin{tabular}{|c|c|c|c|c|c|c|}
\hline type & wt. mean & mean & S. D. & min. & $\max$. & $\mathrm{n}$ \\
\hline rain & 0.20 & 0.39 & 0.47 & 0.02 & 5.40 & 308 \\
\hline rain shower & 0.39 & 1.02 & 0.93 & 0.02 & 3.80 & 46 \\
\hline thundershower & 0.36 & 0.51 & 0.61 & 0.05 & 2.90 & 37 \\
\hline snow & 0.36 & 0.44 & 0.33 & 0.05 & 1.26 & 48 \\
\hline al1 & 0.27 & 0.47 & 0.57 & 0.02 & 5.40 & 439 \\
\hline
\end{tabular}

Table 10d

Variation in Ammonium Concentration (ppm)

by Precipitation Rate

rate (in./hour)

$0.00-0.03$

$0.04-0.10$

$0.11-0.25$

$0.26-0.50$

$\geq 0.51$

al1

\begin{tabular}{l} 
wt. mean \\
\hline 0.66 \\
0.31 \\
0.16 \\
0.18 \\
0.29 \\
0.27
\end{tabular}

S. D.

0.73

0.31

0.13

0.22

0.30

0.57

0.47 
Table 10e

Variation in Ammonium Concentration (ppm)

wind speed

$\left(\mathrm{m} \sec ^{-1}\right)$

$0-2$

$3-5$

6-9

$\geq 10$

a11

by Wind Speed at $108 \mathrm{~m}$

\begin{tabular}{|c|c|c|c|c|c|}
\hline wt. mean & mean & S. D. & min. & $\max$ & $\mathrm{n}$ \\
\hline 0.17 & 0.46 & 0.48 & 0.05 & 1.80 & 21 \\
\hline 0.35 & 0.54 & 0.57 & 0.05 & 3.80 & 166 \\
\hline 0.34 & 0.53 & 0,66 & 0.02 & 5.40 & 164 \\
\hline 0.11 & 0.22 & 0.25 & 0.02 & 1.20 & 88 \\
\hline 0.27 & 0.47 & 0.57 & 0.02 & 5.40 & 439 \\
\hline
\end{tabular}

Table $10 \mathrm{f}$

Variation in Ammonium Concentration (ppm)

by Wind Direction

\begin{tabular}{|c|c|c|c|c|c|c|}
\hline direction & wt. mean & mean & S. D. & min. & $\underline{\max }$. & $\mathrm{n}$ \\
\hline $\mathrm{N}$ & 0.25 & 0.35 & 0.21 & 0.05 & 1.01 & 36 \\
\hline $\mathrm{NE}$ & 0.22 & 0.44 & 0.44 & 0.05 & 1.80 & 58 \\
\hline$E$ & 0.18 & 0.36 & 0.41 & 0.05 & 2.90 & 80 \\
\hline SE & 0.21 & 0.39 & 0.49 & 0.04 & 3.64 & 75 \\
\hline$s$ & 0.25 & 0.60 & 0.77 & 0.02 & 5.40 & 64 \\
\hline SW & 0.48 & 0.83 & 0.82 & 0.02 & 3.80 & 52 \\
\hline W & 0.40 & 0.61 & 0.68 & 0.09 & 2.58 & 23 \\
\hline NW & 0.49 & 0.29 & 0.27 & 0.05 & 1.26 & 51 \\
\hline all & 0.27 & 0.47 & 0.57 & 0.02 & 5.40 & 439 \\
\hline
\end{tabular}

Table $10 \mathrm{~g}$

Variation in Ammonium Concentration (ppm)

by Temperature

\begin{tabular}{|c|c|c|c|c|c|}
\hline temperature $\left({ }^{\circ} \mathrm{F}\right)$ & wt. mean & mean & S. D. & min. & $\max$. \\
\hline $20-29$ & 0.39 & 0.43 & 0.32 & 0.05 & 1.26 \\
\hline $30-39$ & 0.27 & 0.36 & 0.29 & 0.05 & 1.40 \\
\hline $40-49$ & 0.20 & 0.39 & 0.39 & 0.05 & 2.58 \\
\hline $50-59$ & 0.25 & 0.49 & 0.56 & 0.02 & 3.64 \\
\hline $60-69$ & 0.21 & 0.54 & 0.64 & 0.05 & 2.50 \\
\hline $70-79$ & 0.36 & 0.65 & 0.96 & 0.05 & 5.40 \\
\hline $80-89$ & 0.51 & 0.51 & - & 0.51 & 0.51 \\
\hline al1 & 0.27 & 0.47 & 0.57 & 0.02 & 5.40 \\
\hline
\end{tabular}


Table 11a

Variation in Sodium Concentration (ppm)

by Season

season

winter

spring

summer

fall

annual

wt. mean
0.79
0.86
0.38
0.63
0.64

\begin{tabular}{l} 
mean \\
\hline 1.21 \\
1.25 \\
0.87 \\
0.74 \\
1.02
\end{tabular}
S. D.
3.00
1.58
2.90
1.06

2.13

1.02

Table $11 b$

Variation in Sodium Concentration (ppm) by Synoptic Type

type
low
warm front
cold front
occluded front
squall line
hurricane
all

al1

\begin{tabular}{c} 
wt. mean \\
\hline 0.91 \\
0.69 \\
0.38 \\
1.24 \\
0.44 \\
0.75 \\
0.64
\end{tabular}

mean

1.09

1.02

0.59

1.37

1.86

0.82

1.02

S. D.

1. 40

2.13

0.71

1.70

5.44

0.75

2.13

min.

0.07

0.01

0.02

0.09

0.09

0.09

0.01
Table 1lc

Variation in Sodium Concentration ( $\mathrm{ppm}$ )

by Precipitation Type

type
rain
rain shower
thundershower
snow

al1

\begin{tabular}{l} 
wt. mean \\
\hline 0.79 \\
0.73 \\
0.32 \\
0.44 \\
0.64
\end{tabular}

\begin{tabular}{l} 
mean \\
\hline 1.11 \\
1.28 \\
0.44 \\
0.56 \\
1.02
\end{tabular}

S. D.

2.03

min.

3.71

0.51

0.90

2.13

0.01

0.02

0.03

0.06

0.01

Table 11d

Variation in Sodium Concentration ( $\mathrm{ppm}$ ) by Precipitation Rate

\begin{tabular}{|c|c|c|c|c|c|c|}
\hline rate (in./hour) & wt. mean & mean & S. D. & min. & max. & $\mathrm{n}$ \\
\hline $0.00-0.03$ & 0.96 & 1.14 & 2.58 & 0.02 & 24.80 & 156 \\
\hline $0.04-0.10$ & 1.11 & 1.16 & 2.14 & 0.03 & 20.50 & 168 \\
\hline $0.11-0.25$ & 0.57 & 0.59 & 0.74 & 0.01 & 4.38 & 59 \\
\hline $0.26-0.50$ & 0.44 & 0.46 & 0.72 & 0.05 & 2.85 & 23 \\
\hline$\geq 0.51$ & 0.20 & 0.23 & 0.19 & 0.06 & 0.66 & 9 \\
\hline all & 0.64 & 1.02 & 2.13 & 0.01 & 24.80 & 415 \\
\hline
\end{tabular}


Table 1le

Variation in Sodium Concentration (ppm)

by Wind Speed at $108 \mathrm{~m}$

wind speed

$(\mathrm{m} \mathrm{sec}-1)$

$0-2$

$3-5$

$6-9$

$\geq 10$

al1

\begin{tabular}{c} 
wt. mean \\
\hline 0.28 \\
0.28 \\
0.79 \\
1.04 \\
0.64
\end{tabular}

\begin{tabular}{l} 
mean \\
\hline 0.30 \\
0.62 \\
0.99 \\
1.99 \\
1.02
\end{tabular}

min.

0.03

0.01

0.02

0.05

0.01 max.

$$
0.74
$$

24.80

20.50

12.00

24.80

\begin{tabular}{r}
$\mathrm{n}$ \\
\hline 19 \\
158 \\
155 \\
83 \\
415
\end{tabular}

\section{Table 1lf}

Variation in Sodium Concentration (ppm)

by Wind Direction

\begin{tabular}{ccccccc} 
direct1on & wt. mean & mean & S. D. & min. & max. & n \\
\cline { 1 - 1 } N & 0.93 & 1.02 & 1.80 & 0.08 & 7.70 & 33 \\
NE & 0.35 & 0.44 & 0.42 & 0.01 & 2.02 & 52 \\
E & 0.43 & 0.69 & 0.91 & 0.03 & 5.33 & 77 \\
SE & 0.78 & 1.80 & 3.39 & 0.05 & 20.50 & 75 \\
S & 0.84 & 1.05 & 1.23 & 0.02 & 6.00 & 61 \\
SW & 0.79 & 1.20 & 3.58 & 0.08 & 24.80 & 47 \\
W & 0.55 & 0.35 & 0.43 & 0.02 & 1.61 & 19 \\
NW & 0.62 & 0.97 & 1.25 & 0.06 & 5.42 & 51 \\
a11 & 0.64 & 1.02 & 2.13 & 0.01 & 24.80 & 415
\end{tabular}

Table 11g

Variation in Sodium Concentration (ppm)

by Temperature

\begin{tabular}{c} 
temperature $\left({ }^{\circ} \mathrm{F}\right)$ \\
\hline $20-29$ \\
$30-39$ \\
$40-49$ \\
$50-59$ \\
$60-69$ \\
$70-79$ \\
$80-89$ \\
a11
\end{tabular}

\begin{tabular}{c} 
wt. mean \\
\hline 0.38 \\
1.01 \\
0.58 \\
0.95 \\
0.61 \\
0.39 \\
0.09 \\
0.64
\end{tabular}

\begin{tabular}{ccc} 
mean & S. D. & min. \\
\hline 0.41 & 0.41 & 0.06 \\
1.38 & 1.67 & 0.03 \\
0.97 & 2.26 & 0.01 \\
1.15 & 2.24 & 0.02 \\
0.79 & 1.14 & 0.08 \\
1.04 & 3.22 & 0.06 \\
0.09 & - & 0.09 \\
1.02 & 2.13 & 0.01
\end{tabular}

max.

1.54

$\frac{\mathrm{n}}{23}$

7.70

20.50

108

15.40

86

6.00

24.80

0.09

73

59

1

415 




type
low
warm front
cold front
occluded front
squall line
hurricane
all

type

rain

rain shower

thund ershower

snow

a11

\begin{tabular}{|c|c|c|c|c|c|}
\hline wt. mean & mean & S. D. & min. & $\underline{\max }$. & $\mathrm{n}$ \\
\hline 2.18 & 2.95 & 3.98 & 0.30 & $19.00^{\circ}$ & 76 \\
\hline 1.72 & 2.66 & 5.75 & 0.20 & 49.50 & 242 \\
\hline $1 . .02$ & 1.47 & 1.29 & 0.20 & 6.00 & 66 \\
\hline 2.93 & 2.80 & 2.66 & 0.20 & 7.90 & 16 \\
\hline 0.87 & 3.57 & 10.16 & 0.50 & 46.50 & 20 \\
\hline 1.59 & 1.66 & 1.34 & 0.50 & 4.00 & 8 \\
\hline 1.56 & 2.55 & 5.18 & 0.20 & 49.50 & 429 \\
\hline
\end{tabular}

Table 12c

Variation in Chloride Concentration (ppm)

by Precipitation Type

Variation in Chloride Concentration (ppm)

by Synoptic Type 
Table $12 \mathrm{e}$

Variation in Chloride Concentration (ppm)

by Wind Speed at $108 \mathrm{~m}$

wind speed

$(\mathrm{m} \mathrm{sec}-1)$

$0-2$

3-5

6-9

$\geq 10$

a11 direction

$\mathrm{N}$

$\mathrm{NE}$

E

SE

$\mathrm{S}$

SW

$W$

NW

a11

\begin{tabular}{c} 
wt. mean \\
\hline 0.52 \\
0.85 \\
1.93 \\
2.31
\end{tabular}

1.56

\begin{tabular}{l} 
mean \\
\hline 0.78 \\
1.50 \\
2.81 \\
4.36 \\
2.55
\end{tabular}

0.48
3.77
6.49
4.72
5.18

0.20
0.20
0.20
0.20
0.20

Table 12f

Variation in Ch1oride Concentration (ppm) by Wind Direction

\begin{tabular}{c} 
wt. mean \\
\hline 2.12 \\
1.08 \\
1.18 \\
1.91 \\
1.76 \\
1.80 \\
1.61 \\
1.22 \\
1.56
\end{tabular}

\begin{tabular}{cc} 
mean & S. D. \\
\hline 2.31 & 3.74 \\
1.20 & 0.94 \\
2.54 & 5.69 \\
4.35 & 8.19 \\
2.25 & 2.53 \\
2.63 & 6.38 \\
2.20 & 3.96 \\
1.87 & 1.98 \\
2.55 & 5.18
\end{tabular}

D. D.
3.74
0.94
5.69
8.19
2.53
6.38
3.96
1.98
5.18

min.

0.30

0.20

0.20

0.20

0.20

0.20

0.20

0.30

0.20

\begin{tabular}{rr}
\multicolumn{1}{c}{$\max }$. & $\mathrm{n}$ \\
\cline { 3 - 3 } 17.00 & 35 \\
4.00 & 52 \\
46.70 & 80 \\
49.50 & 76 \\
13.10 & 61 \\
46.50 & 52 \\
19.00 & 23 \\
7.90 & 50 \\
49.50 & 429
\end{tabular}

Table $12 \mathrm{~g}$

Variation in Chloride Concentration (ppm) by Temperature

\begin{tabular}{l} 
temperature $\left({ }^{\circ} \mathrm{F}\right)$ \\
\hline $20-29$ \\
$30-39$ \\
$40-49$ \\
$50-59$ \\
$60-69$ \\
$70-79$ \\
$80-89$ \\
a11
\end{tabular}

\begin{tabular}{c} 
wt. mean \\
\hline 1.07 \\
2.14 \\
1.44 \\
2.27 \\
1.67 \\
0.92 \\
0.30 \\
1.56
\end{tabular}

\begin{tabular}{c} 
mean \\
\hline 1.00 \\
2.89 \\
2.69 \\
3.08 \\
2.31 \\
2.17 \\
0.30 \\
2.55
\end{tabular}

S. D.

1.16

min.

0.30

3.22

0.20

0.20

6.76

0.20

3.14

0.50

6.08

0.50

$-$

0.30

5.18

0.20
max.
6.00
17.00
49.50
37.80
18.50
46.50
0.30
49.50

\begin{tabular}{r}
$\mathrm{n}$ \\
\hline 26 \\
64 \\
116 \\
87 \\
77 \\
52 \\
1 \\
429
\end{tabular}


Table 13a

Variation in Nitrate/Sulfate Ratio

by Season

season
winter
spring
summer
fall
annual wt. mean

0.10

0.09

0.07

0.07

0.08

mean
0.19
0.12
0.08
0.12
0.13

\begin{tabular}{l} 
S.D. \\
\hline 0.18 \\
0.12 \\
0.06 \\
0.09 \\
0.12
\end{tabular}

min.

0.01

0.00

0.01

0.01

0.00
max.
1.05
0.91
0.29
0.62
1.05

$\frac{\mathrm{n}}{94}$

134

70

125

423

Table 13b

Variation in Nitrate/Sulfate Ratio

by Synoptic Type

type
low
warm front
cold front
occluded front
squall line
hurricane
all

type
rain
rain shower
thundershower
snow
all

rate (in./hour)
$0.00-0.03$
$0.04-0.10$
$0.11-0.25$
$0.26-0.50$
20.51
al1

\begin{tabular}{c} 
wt. mean \\
\hline 0.10 \\
0.07 \\
0.11 \\
0.12 \\
0.09 \\
0.02 \\
0.08
\end{tabular}

\begin{tabular}{cc} 
mean & S.D. \\
\hline 0.14 & 0.14 \\
0.12 & 0.12 \\
0.15 & 0.12 \\
0.12 & 0.11 \\
0.14 & 0.09 \\
0.02 & 0.01 \\
0.13 & 0.12
\end{tabular}

Table $13 \mathrm{c}$

Varlation In Nitrate/Sulfate Ratio

by Precipitation 'Iype

\begin{tabular}{l} 
wt. mean \\
\hline 0.07 \\
0.09 \\
0.08 \\
0.23 \\
0.08
\end{tabular}

mean
0.10
0.17
0.11
0.27
0.13

S. D

0.08

min.

$\frac{\text { min. }}{0.01}$

0.00

0.02

0.02

0.04

0.01

0.00

0.08

0.20

0.12

0.01

0.01

0.03

0.00

Table 13d

Variation in Nitrate/Sulfate Ratio

by Precipitation Rate

\begin{tabular}{c} 
wt. mean \\
\hline 0.18 \\
0.12 \\
0.06 \\
0.05 \\
0.06 \\
0.08
\end{tabular}

$\frac{\text { mean }}{0.18}$
0.12
0.06
0.05
0.06
0.13

\begin{tabular}{l} 
S. D. \\
\hline 0.16 \\
0.09 \\
0.05 \\
0.05 \\
0.07 \\
0.12
\end{tabular}

\begin{tabular}{l} 
min. \\
\hline 0.01 \\
0.00 \\
0.01 \\
0.01 \\
0.01 \\
0.00
\end{tabular}

max.

1.05

0.50

0.21

0.19

0.23

$\begin{array}{lr}\text { max. } & \mathrm{n} \\ 0.62 & 74 \\ 1.05 & 242 \\ 0.91 & 66 \\ 0.45 & 15 \\ 0.32 & 17 \\ 0.04 & 8 \\ 1.05 & 423\end{array}$

$\begin{array}{lr}\max . & \mathrm{n} \\ 0.62 & 297 \\ 0.91 & 42 \\ 0.33 & 37 \\ 1.05 & 47 \\ 1.05 & 423\end{array}$


Table 13e

Variation in Nitrate/Sulfate Ratio

by Wind Speed at $108 \mathrm{~m}$

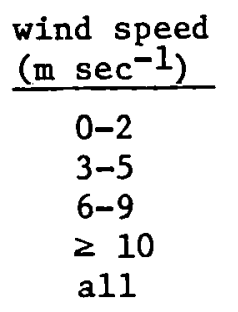

direction

N

NE

E

SE

$\mathrm{S}$

SW

W

NW

all

\begin{tabular}{|c|c|c|c|c|c|}
\hline wt. mean & mean & S.D. & min. & max. & $\mathrm{n}$ \\
\hline 0.11 & 0.22 & 0.17 & 0.01 & 0.62 & 19 \\
\hline 0.09 & 0.14 & 0.11 & 0.01 & 0.55 & 157 \\
\hline 0.11 & 0.14 & 0.13 & 0.00 & 1.05 & 161 \\
\hline 0.04 & 0.08 & 0.10 & 0.01 & 0.62 & 86 \\
\hline 0.08 & 0.13 & 0.1 .2 & 0.00 & 1.05 & 423 \\
\hline
\end{tabular}

Table 13f

Variation in Nitrate/Sulfate Ratio

by Wind Direction

\begin{tabular}{cccccc} 
wt. mean & mean & S. D. & min. & max. & n \\
\cline { 1 - 1 } 0.12 & 0.17 & 0.13 & 0.01 & 0.55 & 34 \\
0.09 & 0.16 & 0.16 & 0.01 & 0.72 & 56 \\
0.05 & 0.10 & 0.13 & 0.01 & 1.05 & 77 \\
0.06 & 0.09 & 0.09 & 0.00 & 0.47 & 74 \\
0.07 & 0.11 & 0.11 & 0.02 & 0.62 & 62 \\
0.14 & 0.18 & 0.13 & 0.02 & 0.91 & 50 \\
0.17 & 0.15 & 0.05 & 0.08 & 0.25 & 21 \\
0.11 & 0.14 & 0.11 & 0.03 & 0.62 & 49 \\
0.08 & 0.13 & 0.12 & 0.00 & 1.05 & 423
\end{tabular}

Table 13g

Variation in Nitrate/Sulfate Ratio

by Temperature

temperature $\left({ }^{\circ} \mathrm{F}\right)$

20-29

30-39

40-49

50-59

60-69

$70-79$

80-89

a11

\begin{tabular}{l} 
wt. mean \\
\hline 0.26 \\
0.11 \\
0.08 \\
0.07 \\
0.07 \\
0.06 \\
0.19 \\
0.08
\end{tabular}

\begin{tabular}{cc} 
S. D. & min. \\
\hline 0.18 & 0.07 \\
0.16 & 0.01 \\
0.10 & 0.00 \\
0.12 & 0.01 \\
0.07 & 0.01 \\
0.06 & 0.01 \\
- & 0.19 \\
0.12 & 0.00
\end{tabular}

max.

0.72

1.05

0.62

0.91

0.32

0.29

0.19

1.05

\begin{tabular}{r}
\multicolumn{1}{c}{$\mathbf{2}$} \\
26 \\
65 \\
118 \\
84 \\
71 \\
58 \\
1 \\
423
\end{tabular}

423 
Table 14a

Variation in Ammonium/Sulfate Ratio

by Season

\begin{abstract}
season
winter

spring

summer

fal1
\end{abstract}

annual

\begin{tabular}{l} 
wt. mean \\
\hline 0.06 \\
0.08 \\
0.05 \\
0.08 \\
0.07
\end{tabular}

\begin{tabular}{l} 
mean \\
\hline 0.10 \\
0.10 \\
0.07 \\
0.14 \\
0.11
\end{tabular}

S. D

0.07

0.12

0.06

0.13

0.11

Table $14 \mathrm{~b}$

Variation in Ammonium/Sulfate Ratio. by Synoptic Type

type
low
warm front
cold front
occluded front
squall line
hurricane
all

type
rain
rain shower
thundershower
snow
al1

Table 14c

Variation in Ammonium/Sulfate Ratio

by Precipitation Type

\begin{tabular}{c} 
wt. mean \\
\hline 0.07 \\
0.07 \\
0.07 \\
0.06 \\
0.07 \\
0.03 \\
0.07
\end{tabular}

\begin{tabular}{|c|c|}
\hline mean & S. D. \\
\hline 0.10 & 0.07 \\
\hline 0.11 & 0.10 \\
\hline 0.14 & 0.16 \\
\hline 0.06 & 0.05 \\
\hline 0.10 & 0.08 \\
\hline 0.03 & 0.02 \\
\hline 0.11 & 0.11 \\
\hline
\end{tabular}

\begin{tabular}{l} 
min. \\
\hline 0.01 \\
0.01 \\
0.01 \\
0.02 \\
0.03 \\
0.02 \\
0.01
\end{tabular}

max.

0.33

0.94

1.07

0.20

0.32

0.06

1.07

\begin{tabular}{r}
$\mathrm{n}$ \\
\hline 93 \\
134 \\
72 \\
130 \\
429
\end{tabular}

429

\begin{tabular}{lllllr} 
wt. mean & mean & S. D. & min. & max. & n \\
\cline { 1 - 2 } 0.07 & 0.10 & 0.10 & 0.01 & 0.94 & 302 \\
0.07 & 0.17 & 0.19 & 0.01 & 1.07 & 44 \\
0.06 & 0.07 & 0.06 & 0.02 & 0.33 & 37 \\
0.10 & 0.11 & 0.07 & 0.03 & 0.28 & 46 \\
0.07 & 0.11 & 0.11 & 0.01 & 1.07 & 429
\end{tabular}

Table 14d

Varlation in Ammonium/Sulfate Ratio

by Precipitation Rate

\begin{tabular}{lcccccr} 
rate (in./hour) & wt. mean & mean & S. D. & min. & max. & n \\
\cline { 1 - 1 } $0.00-0.03$ & 0.14 & 0.16 & 0.14 & 0.01 & 1.07 & 169 \\
$0.04-0.10$ & 0.09 & 0.09 & 0.06 & 0.01 & 0.56 & 169 \\
$0.11-0.25$ & 0.06 & 0.06 & 0.04 & 0.01 & 0.18 & 59 \\
$0.26-0.50$ & 0.04 & 0.05 & 0.04 & 0.02 & 0.17 & 23 \\
20.51 & 0.05 & 0.05 & 0.04 & 0.01 & 0.11 & 9 \\
al1 & 0.07 & 0.11 & 0.11 & 0.01 & 1.07 & 429
\end{tabular}


Table 14e

Variation in Ammonium/Sulfate Ratio by Wind Speed at $108 \mathrm{~m}$

wind speed

$\left(\mathrm{m} \mathrm{sec}^{-1}\right)$

$0-2$

$3-5$

6-9

$\geq 10$

al1 direction

$\mathrm{N}$

NE

E

SE

$S$

SW

W

NW

al1

1

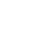

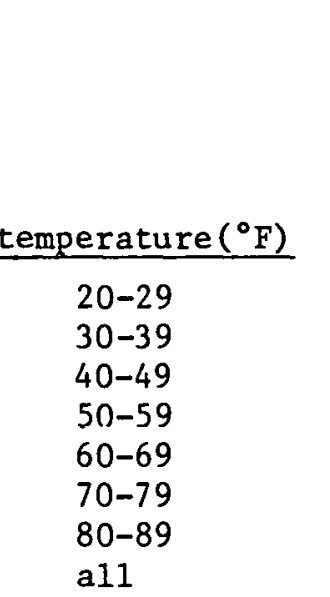

\section{temperature $\left({ }^{\circ} \mathrm{F}\right)$}

\begin{tabular}{l} 
wt. mean \\
\hline 0.06 \\
0.07 \\
0.09 \\
0.05 \\
0.07
\end{tabular}

\begin{tabular}{|c|c|}
\hline nean & S. D. \\
\hline 0.11 & 0.07 \\
\hline 0.11 & 0.11 \\
\hline 0.12 & 0.11 \\
\hline 0.08 & 0.08 \\
\hline 0.11 & 0.11 \\
\hline
\end{tabular}

Table $14 f$

Variation in Ammonium/Sulfate Ratio by Wind Direction

\begin{tabular}{l} 
wt. mean \\
\hline 0.09 \\
0.07 \\
0.06 \\
0.05 \\
0.08 \\
0.09 \\
0.09 \\
0.08 \\
0.07
\end{tabular}

min. 0.03 0.02 0.02 0.01 0.01 0.05 0.02 0.01 0.02
0.04

0.11 min.
0.02
0.01
0.01
0.01
0.01 max.

0.29

0.94

1.07

0.56

1.07

\begin{tabular}{r} 
n \\
\hline 21 \\
159 \\
162 \\
87 \\
429
\end{tabular}

429

Table $14 \mathrm{~g}$

Variation in Ammonium/Sulfate Ratio

by Temperature

\begin{tabular}{lccc} 
mean & & S. D. & min. \\
\hline 0.11 & 0.06 & 0.03 \\
0.09 & 0.06 & 0.02 \\
0.10 & 0.09 & 0.02 \\
0.12 & 0.13 & 0.01 \\
0.12 & 0.16 & 0.01 \\
0.09 & 0.08 & 0.01 \\
0.10 & - & 0.10 \\
0.11 & 0.11 & 0.01
\end{tabular}

max.

max.

0.26

0.33

0.28

0.41

0.94

1.07

0.81

0.19

1.07

\begin{tabular}{r}
$\mathrm{n}$ \\
\hline 35 \\
56 \\
79 \\
75 \\
63 \\
51 \\
21 \\
49 \\
429
\end{tabular}

\begin{tabular}{l} 
wt. mean \\
\hline 0.10 \\
0.08 \\
0.07 \\
0.07 \\
0.06 \\
0.05 \\
0.10 \\
0.07
\end{tabular}

0.11
0.27

0.28

0.81

1.07

0.94

0.44

0.10

1.07

\begin{tabular}{r}
\multicolumn{1}{c}{$\mathbf{n}$} \\
26 \\
65 \\
116 \\
89 \\
75 \\
58 \\
1 \\
429
\end{tabular}


Table 15a

Variation in Ammonium/Nitrate Ratio

by Season

season
winter
spring
summer
fall

annual

\begin{tabular}{|c|c|c|c|c|c|}
\hline t. mean & mean & S. D. & min. & $\max$. & $\mathbf{n}$ \\
\hline 1.42 & 1.05 & 1.52 & 0.15 & 13.50 & 92 \\
\hline 1.37 & 1.54 & 2.67 & 0.09 & 21.00 & 136 \\
\hline 1.00 & 1.16 & 0.92 & 0.21 & 5.25 & 71 \\
\hline 1.76 & 1.58 & 2.04 & 0.22 & 20.00 & 125 \\
\hline 1.34 & 1.38 & 2.05 & 0.09 & 21.00 & 424 \\
\hline
\end{tabular}

Table 15b

Variation in Ammonium/Nitrate Ratio

by Synoptic Type

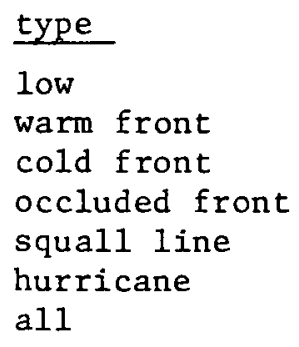

type

rain

rain shower

thundershower

snow

al1

\begin{tabular}{c} 
wt. mean \\
\hline 1.24 \\
1.61 \\
0.77 \\
1.12 \\
0.92 \\
1.39 \\
1.34
\end{tabular}

\begin{tabular}{l} 
mean \\
\hline 1.27 \\
1.55 \\
1.09 \\
1.20 \\
0.80 \\
1.57 \\
1.38
\end{tabular}

S. D.

1.44

2.47

1.18

1.55

0.45

0.66

2.05

Table 15c

Variation in Ammonium/Nitrate Ratio

by Precipitation Type

\begin{tabular}{|c|c|c|c|c|c|}
\hline wt mean & mean & S. D. & min. & $\max$. & $\mathrm{n}$ \\
\hline 1.52 & 1.49 & 2.16 & 0.09 & 21.00 & 296 \\
\hline 0.98 & 1.58 & 1.99 & 0.23 & 8.90 & 44 \\
\hline 1.14 & 1.26 & 2.16 & 0.12 & 13.50 & 37 \\
\hline 0.71 & 0.58 & 0.57 & 0.15 & 3.71 & 47 \\
\hline 1.34 & 1.38 & 2.05 & 0.09 & 21.00 & 424 \\
\hline
\end{tabular}

\section{Table 15d}

Variation in Ammonium/Nitrate Ratio by Precipitation Rate

rate (in./
$0.00-0.03$
$0.04-0.10$
$0.11-0.25$
$0.26-0.50$
20.51
al1

\begin{tabular}{|c|c|c|c|c|c|}
\hline wt. mean & mean & S. D. & mir. & $\underline{\max }$. & $\mathrm{n}$ \\
\hline 1.35 & 1.38 & 1.60 & 0.16 & 10.00 & 164 \\
\hline 1.34 & 1.40 & 2.77 & 0.09 & 21.00 & 169 \\
\hline 1.34 & 1.32 & 0.85 & 0.12 & 4.00 & 59 \\
\hline 1.43 & 1.43 & 0.78 & 0.42 & 2.50 & 23 \\
\hline 1.22 & 1.38 & 0.86 & 0.49 & 2.50 & 9 \\
\hline 1.34 & 1.38 & 2.05 & 0.09 & 21.00 & 424 \\
\hline
\end{tabular}


Table 15e

Variation in Ammonium/Nitrate Ratio

by Wind Speed at $108 \mathrm{~m}$

wind speed

$\left(\mathrm{m} \sec ^{-1}\right)$

$0-2$

$3-5$

$6-9$

2. 10

al1

\begin{tabular}{l} 
wt. mean \\
\hline 1.00 \\
1.14 \\
1.18 \\
1.76 \\
1.34
\end{tabular}

mean
1.00
1.28
1.23
1.93
1.38

\begin{tabular}{l} 
S. D. \\
\hline 1.64 \\
1.66 \\
1.83 \\
2.89 \\
2.05
\end{tabular}

$\frac{\text { min. }}{0.16}$

$\underline{\max }$.

7.50

$0.12 \quad 13.50$

0.21

0.09

0.09

21.00

20.00

21.00

\begin{tabular}{r}
$\mathrm{n}$ \\
\hline 19 \\
158 \\
160 \\
87 \\
424
\end{tabular}

Table 15f

Variation in Ammonium/Nitrate Ratio

by Wind Direction

\begin{tabular}{|c|c|c|c|c|c|c|}
\hline direction & wt. mean & mean & S. D. & min. & $\underline{\max }$. & $\mathbf{n}$ \\
\hline $\mathrm{N}$ & 1.14 & 0.94 & 0.55 & 0.22 & 2.50 & 35 \\
\hline NE & 1.20 & 1.40 & 2.19 & 0.12 & 13.50 & 56 \\
\hline$E$ & 1.56 & 1.46 & 1.23 & 0.23 & 7.50 & 78 \\
\hline $\mathrm{SE}$ & 1.52 & 1.90 & 3.21 & 0.21 & 21.00 & 74 \\
\hline $\mathrm{S}$ & 1.51 & 1.96 & 2.69 & 0.22 & 20.00 & 62 \\
\hline SW & 0.70 & 0.79 & 0.51 & 0.23 & 3.18 & 48 \\
\hline$W$ & 0.61 & 1.05 & 1.87 & 0.23 & 8.90 & 20 \\
\hline NW & 0.97 & 0.77 & 0.60 & 0.09 & 3.71 & 51 \\
\hline all & 1.34 & 1.38 & 2.05 & 0.09 & 21.00 & 424 \\
\hline & & Tab & $5 g$ & & & \\
\hline & Variation & in Amm & $\mathrm{m} / \mathrm{Nitr}$ & Ratio & & \\
\hline & & y Tem & ture & & & \\
\hline temperature $\left({ }^{\circ} \mathrm{F}\right)$ & wt. mean & mean & S. D. & min. & $\underline{\max }$. & $\mathrm{n}$ \\
\hline $20-29$ & 0.55 & 0.47 & 0.58 & 0.15 & 3.18 & 27 \\
\hline $30-39$ & 1.40 & 1.13 & 1.69 & 0.09 & 13.50 & 66 \\
\hline $40-49$ & 1.49 & 1.46 & 2.75 & 0.12 & 21.00 & 115 \\
\hline $50-59$ & 1.30 & 1.38 & 1.24 & 0.22 & 8.64 & 85 \\
\hline $60-69$ & 1.59 & 1.84 & 2.60 & 0.21 & 20.00 & 72 \\
\hline $70-79$ & 1.12 & 1.37 & 1.02 & 0.28 & 5.25 & 58 \\
\hline $80-89$ & 0.52 & 0.52 & - & 0.52 & 0.52 & 1 \\
\hline all & 1.34 & 1.38 & 2.05 & 0.09 & 21.00 & 424 \\
\hline
\end{tabular}


Tablè 16a

Variation in Sulfate/Sodium Ratio

by Season

season
winter
spring
summer
fall
annual

\begin{tabular}{l} 
Wt. mean \\
\hline 15.65 \\
18.61 \\
32.17 \\
13.04 \\
21.48
\end{tabular}

21.48

\begin{tabular}{|c|c|}
\hline mean & S. D. \\
\hline 14.01 & 13.27 \\
\hline 15.21 & 28.04 \\
\hline 23.92 & 32.26 \\
\hline 14.28 & $17: 41$ \\
\hline 16.17 & 23.64 \\
\hline
\end{tabular}

Table 16b

Variation in Sulfate/Sodium Ratio by Synoptic Type

$\frac{\text { type }}{\text { low }}$
warm front
cold front
occluded front
squall line
hurricane
all

type
rain
rain shower
thundershower
snow
all

\begin{tabular}{l} 
wt. mean \\
\hline 9.70 \\
17.22 \\
45.11 \\
4.99 \\
25.57 \\
11.91 \\
21.48
\end{tabular}

Table 16c

Variation in Sulfate/Sodium Ratio

by Precipitation Type

\begin{tabular}{|c|c|c|c|c|}
\hline mean & S. D. & min. & $\max$. & $\mathrm{n}$ \\
\hline 9.18 & 10.33 & 0.57 & 57.14 & 73 \\
\hline 16.08 & 23.41 & 0.34 & 200.00 & 232 \\
\hline 24.34 & 29.88 & 0.93 & 160.00 & 61 \\
\hline 6.12 & 6.33 & 0.43 & 22.22 & 15 \\
\hline 28.56 & 39.90 & 0.56 & 164.57 & 17 \\
\hline 10.91 & 11.88 & 1.18 & 27.67 & 8 \\
\hline 16.17 & 23.64 & 0.34 & 200.00 & 407 \\
\hline
\end{tabular}

$\begin{array}{llrlrr}\text { wt. mean } & \text { mean } & \text { S. D. } & \text { min. } & \text { max. } & \frac{\mathrm{n}}{291} \\ 15.72 & 13.74 & 21.60 & 0.34 & 200.00 & 291 \\ 11.98 & 25.82 & 39.63 & 0.56 & 164.57 & 41 \\ 38.29 & 26.53 & 21.17 & 1.12 & 84.67 & 36 \\ 16.38 & 14.55 & 9.78 & 1.45 & 33.33 & 39 \\ 21.48 & 16.17 & 23.64 & 0.34 & 200.00 & 407\end{array}$

Table 16d

Variation in Sulfate/Sodium Ratio

by Precipitation Rate

\begin{tabular}{|c|c|c|c|c|c|c|}
\hline rate (in./hour) & wt. mean & mean & S. D. & min. & $\underline{\max }$. & $\mathrm{n}$ \\
\hline $0.00-0.03$ & 16.66 & 16.74 & 26.52 & 0.46 & 164.57 & 150 \\
\hline $0.04-0.10$ & 12.90 & 13.52 & 16.78 & 0.34 & 117.50 & 166 \\
\hline $0.11-0.25$ & 18.79 & 18.68 & 32.88 & 0.71 & 200.00 & 59 \\
\hline $0.26-0.50$ & 20.21 & 20.06 & 13.79 & 1.31 & 58.44 & 23 \\
\hline 20.51 & 39.41 & 28.83 & 26.13 & 3.05 & 84.67 & 9 \\
\hline a11 & 21.48 & 16.17 & 23.64 & 0.34 & 200.00 & 407 \\
\hline
\end{tabular}


Table 16e

Variation in Sulfate/Sodium Ratio

by Wind Speed at $108 \mathrm{~m}$

wind speed

$(\mathrm{m} \mathrm{sec}-1)$

$0-2$

$3-5$

6-9

$\geq 10$

a11 wt. mean

17.06

37.04

12.16

9.84

21.48

$\begin{array}{rr}\text { mean } & \text { S. D. } \\ 18.83 & 16.32 \\ 26.05 & 31.58 \\ 11.91 & 16.05 \\ 5.29 & 7.70 \\ 16.17 & 23.64\end{array}$

16.17 min.

4.08

0.56

0.68

0.34

0.34 max.

73.33

200.00

160.00

200.00

$\frac{\mathrm{n}}{19}$
152
153
83
407

407

Table $16 \mathrm{f}$

Variation in Sulfate/Sodium Ratio

by Wind Direction

direction

$\mathrm{N}$

$\mathrm{NE}$

E

SE

$S$

$\mathrm{SW}$

W

$\mathrm{NW}$

al1 wt. mean

9.69

32.29

16.34

30.36

9.29

18.36

16.46

25.38

21.48

$\begin{array}{rr}\frac{\text { mean }}{9.73} & \frac{\text { S. D. }}{9.22} \\ 21.05 & 32.47 \\ 14.34 & 14.77 \\ 17.77 & 31.57 \\ 10.35 & 15.21 \\ 16.50 & 15.48 \\ 29.00 & 35.36 \\ 17.14 & 23.41 \\ 16.17 & 23.64\end{array}$

Table $16 \mathrm{~g}$

Variation in Sulfate/Sodium Ratio

by Temperature

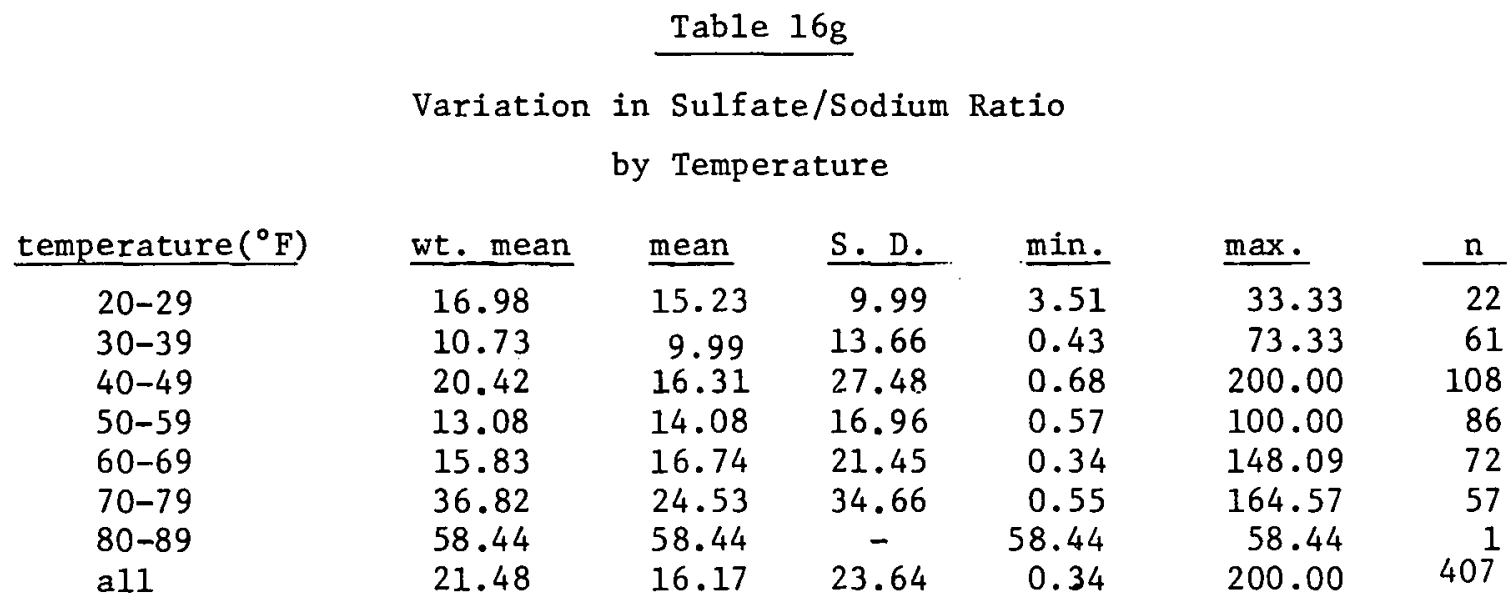

\begin{tabular}{|c|c|c|c|c|c|c|}
\hline \multirow[b]{2}{*}{ temperature $\left({ }^{\circ} \mathrm{F}\right)$} & \multicolumn{4}{|c|}{$\begin{array}{c}\text { Variation in Sulfate/Sodium Ratio } \\
\text { by Temperature }\end{array}$} & \multirow[b]{2}{*}{$\underline{\max }}$. & \multirow[b]{2}{*}{$\underline{\mathrm{n}}$} \\
\hline & wt. mean & mean & S. D. & min. & & \\
\hline $20-29$ & 16.98 & 15.23 & 9.99 & 3.51 & 33.33 & 22 \\
\hline $30-39$ & 10.73 & 9.99 & 13.66 & 0.43 & 73.33 & 61 \\
\hline $40-49$ & 20.42 & 16.31 & 27.48 & 0.68 & 200.00 & 108 \\
\hline $50-59$ & 13.08 & 14.08 & 16.96 & 0.57 & 100.00 & 86 \\
\hline $60-69$ & 15.83 & 16.74 & 21.45 & 0.34 & 148.09 & 72 \\
\hline $70-79$ & 36.82 & 24.53 & 34.66 & 0.55 & 164.57 & 57 \\
\hline $80-89$ & 58.44 & 58.44 & - & 58.44 & 58.44 & 1 \\
\hline a11 & 21.48 & 16.17 & 23.64 & 0.34 & 200.00 & $40 \overline{7}$ \\
\hline
\end{tabular}

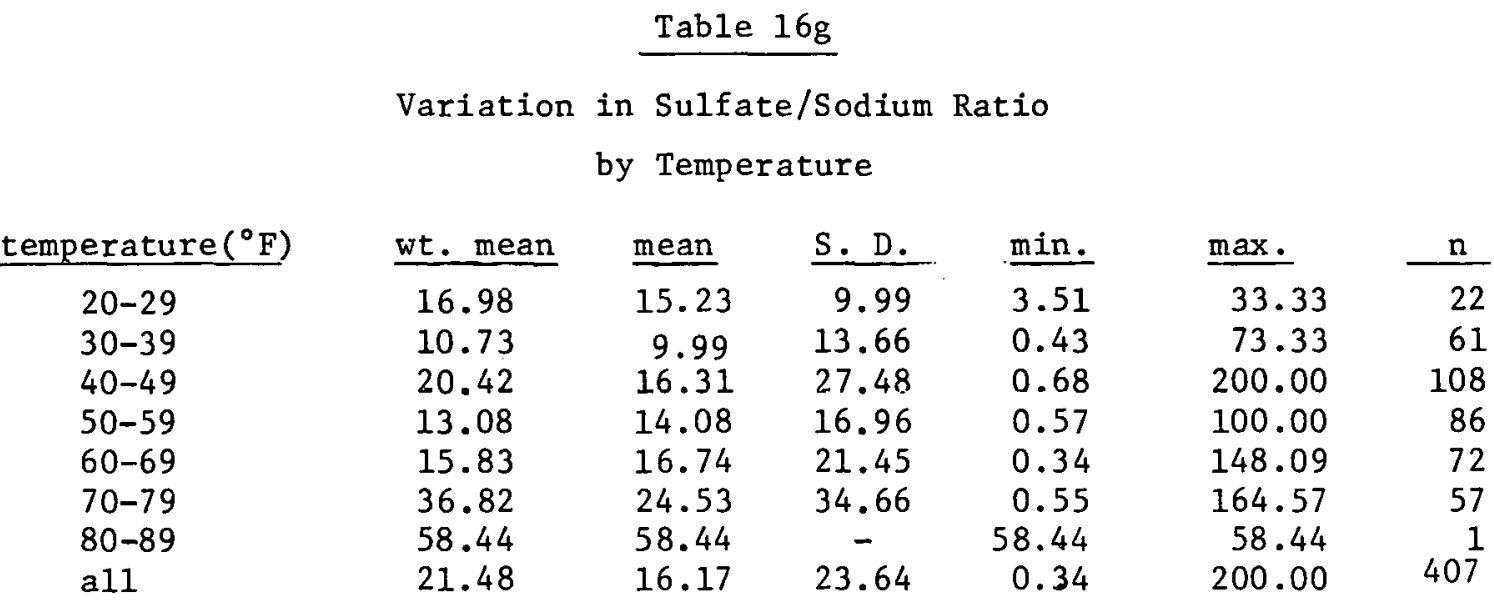

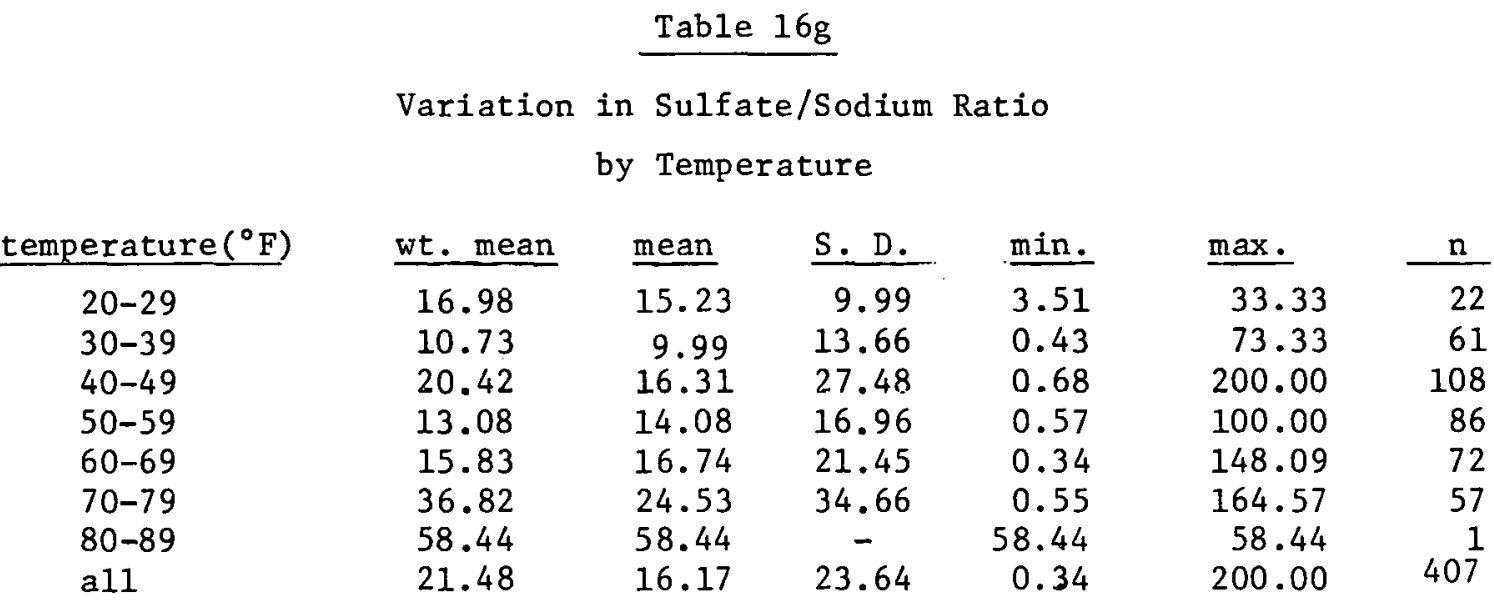

\begin{tabular}{l} 
min. \\
\hline 0.68 \\
2.13 \\
0.91 \\
0.57 \\
0.34 \\
0.57 \\
1.85 \\
0.43 \\
0.34
\end{tabular}

max.

36.68

200.00

73.33

164.57

100.00

60.25

160.00

148.09

200.00

\begin{tabular}{l}
$\mathrm{n}$ \\
\hline 30 \\
52 \\
76 \\
74 \\
60 \\
47 \\
19 \\
49 \\
407
\end{tabular}

49
407 
Table 17a

Variation in Sulfate/Chloride Ratio

by Season

season

winter

spring

summer

fall

annual wt. mean

4.80
4.58
8.40
2.93
5.58

4.80

5.58

mean
4.28
4.18
6.65
3.35
4.37

\begin{tabular}{l} 
S. D. \\
\hline 3.80 \\
4.77 \\
5.91 \\
2.76 \\
4.41
\end{tabular}

min.

0.25

0.11

0.30

0.15

0.11

Table 17b

Variation in Sulfate/Chloride Ratio

by Synoptic Trpe

(2)

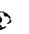



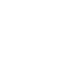

type
low
warm front
cold front
occluded front
squall line
hurricane
all

type.
rain
rain shower
thundershower
snow
all

\begin{tabular}{c} 
wt. mean \\
\hline 2.92 \\
4.19 \\
9.27 \\
2.15 \\
10.13 \\
2.92 \\
5.58
\end{tabular}

mean
3.13
4.06
6.27
2.75
8.08
2.76
4.37

\begin{tabular}{l} 
S. D. \\
\hline 3.57 \\
3.98 \\
4.78 \\
2.71 \\
7.94 \\
1.97 \\
4.41
\end{tabular}

min. 0.11

0.15

0.40

0.25

0.30

0.62

0.11

Table $17 \mathrm{c}$

Variation in Sulfate/Chloride Ratio

by Precipitation Type

\begin{tabular}{c} 
wt. mean \\
\hline 3.70 \\
4.47 \\
10.29 \\
5.03 \\
5.58
\end{tabular}

\begin{tabular}{|c|c|}
\hline mean & S. D. \\
\hline 3.54 & 3.67 \\
\hline 6.10 & 6.05 \\
\hline 8.63 & 5.81 \\
\hline 4.77 & 3.25 \\
\hline 4.37 & 4.41 \\
\hline
\end{tabular}

min.

0.11

0.30

0.49

0.29

0.11

Table 17d

Variation in Sulfate/Chiloride Ratio

by Precipitation Rate

rate (in./hour)
$0.00-0.03$
$0.04-0.10$
$0.11-0.25$
$0.26-0.50$
$\geq 0.51$
all

\begin{tabular}{c} 
wt. mean \\
\hline 4.66 \\
3.82 \\
3.74 \\
5.78 \\
10.36 \\
5.58
\end{tabular}

mean
4.50
4.03
3.76
5.80
8.57
4.37

\begin{tabular}{l} 
S. D. \\
\hline 4.81 \\
4.16 \\
3.20 \\
4.48 \\
5.80 \\
4.41
\end{tabular}

\begin{tabular}{l} 
min. \\
\hline 0.11 \\
0.15 \\
0.35 \\
0.54 \\
1.18 \\
0.11
\end{tabular}

$\frac{\max .}{32.91}$
24.10
13.50
17.53
19.80
32.91

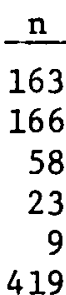

\begin{tabular}{r}
\multicolumn{1}{c}{$\mathrm{n}$} \\
92 \\
132 \\
70 \\
125 \\
419
\end{tabular}

13.20

419 
Table 17e

Variation in Sulfate/Chleride Ratio

wind speed

by Wind Speed at $108 \mathrm{~m}$

(m $\left.\sec ^{-1}\right)$

$0-2$

$3-5$

6-9

$\geq 10$

al1

\begin{tabular}{c} 
wt. mean \\
\hline 5.52 \\
8.39 \\
4.01 \\
3.30 \\
5.58
\end{tabular}

mean

5.13

6.14

3.89

2.01

4.37
S. D.

2.53

5.23

3.79

2.60

4.41 min.

2.23

0.30

0.22

0.11

0.11 max.

11.00

32.91

21.92

10.00

32.91

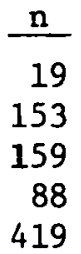

419

Table 17f

Variation in Sulfate/Chloride Ratio

by Wind Direction

\begin{tabular}{|c|c|c|c|c|c|c|}
\hline direction & wt. mean & mean & S. D. & min. & $\underline{\max }$. & $n$ \\
\hline $\mathrm{N}$ & 3.07 & 3.23 & 2.80 & 0.31 & 13.20 & 32 \\
\hline $\mathrm{NE}$ & 4.36 & 4.51 & 4.74 & 0.71 & 28.50 & 52 \\
\hline E & 4.50 & 4.10 & 3.57 & 0.22 & 20.95 & 76 \\
\hline SE & 6.56 & 4.31 & 5.29 & 0.25 & 32.91 & 75 \\
\hline $\mathrm{S}$ & 3.42 & 3.67 & 3.47 & 0.15 & 16.15 & 61 \\
\hline SW & 5.94 & 5.55 & 5.28 & 0.30 & 24.10 & 52 \\
\hline $\mathrm{W}$ & 5.08 & 4.81 & 4.08 & 0.11 & 15.99 & 23 \\
\hline $\mathrm{NW}$ & 10.07 & 4.89 & 4.74 & 0.25 & 19.80 & 48 \\
\hline all & 5.58 & 4.37 & 4.41 & 0.11 & 32.91 & 419 \\
\hline
\end{tabular}

Table $17 \mathrm{~g}$

Variation in Sulfate/ Chloride Ratio

by Temperature

temperature $\left({ }^{\circ} \mathrm{F}\right)$

20-29

30-39

40-49

50-59

60-69

$70-79$

80-89

a11

\begin{tabular}{c} 
wt. mean \\
\hline 5.82 \\
3.44 \\
4.87 \\
3.50 \\
4.26 \\
9.21 \\
17.53 \\
5.58
\end{tabular}

mean

5.70

3.04

4.14

3.55

4.38

6.71

17.53

4.37

S. D.

3.63

3.33

3.82

4.10

4.10

6.22

$4 . \overline{41}$

4.41

17.53

0.11

min.

0.40

0.25

0.22

0.11

0.15

0.29

max.

18.00

20.95

28.50

24.10

21.92

32.91

17.53

32.91

\begin{tabular}{r} 
n \\
\hline 25 \\
60 \\
116 \\
87 \\
74 \\
56 \\
1 \\
419
\end{tabular}




\begin{tabular}{|c|c|c|c|c|c|c|}
\hline \multirow[b]{2}{*}{ season } & \multicolumn{4}{|c|}{$\begin{array}{c}\text { Variation in Chloride/Sodium Ratio } \\
\text { by Season }\end{array}$} & \multirow[b]{2}{*}{$\underline{\max }}$. & \multirow[b]{2}{*}{$\mathrm{n}$} \\
\hline & wt. mean & mean & $\underline{\text { S. D. }}$ & min. & & \\
\hline $\begin{array}{l}\text { winter } \\
\text { spring } \\
\text { summer } \\
\text { fall } \\
\text { annual }\end{array}$ & $\begin{array}{l}3.16 \\
3.63 \\
3.96 \\
4.19 \\
3.76\end{array}$ & $\begin{array}{l}3.33 \\
3.29 \\
3.48 \\
4.00 \\
3.55\end{array}$ & $\begin{array}{l}2.36 \\
5.32 \\
3.29 \\
3.03 \\
3.81\end{array}$ & $\begin{array}{l}0.19 \\
0.81 \\
1.28 \\
1.32 \\
0.19\end{array}$ & $\begin{array}{l}17.50 \\
50.00 \\
25.50 \\
20.83 \\
50.00\end{array}$ & $\begin{array}{r}88 \\
123 \\
71 \\
124 \\
406\end{array}$ \\
\hline \multicolumn{7}{|c|}{ Table $18 \mathrm{~b}$} \\
\hline \multicolumn{7}{|c|}{$\begin{array}{c}\text { Variation in Chloride/Sodium Ratio } \\
\text { by Synoptic Type }\end{array}$} \\
\hline type & wt. mean & mean & S.D. & min. & $\underline{\max }$. & $\mathrm{n}$ \\
\hline $\begin{array}{l}\text { low } \\
\text { warm front } \\
\text { cold front } \\
\text { occluded front } \\
\text { squall line } \\
\text { hurricane } \\
\text { all }\end{array}$ & $\begin{array}{l}3.33 \\
4.00 \\
4.38 \\
3.75 \\
2.41 \\
3.20 \\
3.76\end{array}$ & $\begin{array}{l}3.24 \\
3.67 \\
3.90 \\
3.04 \\
2.83 \\
3.03 \\
3.55\end{array}$ & $\begin{array}{l}2.73 \\
4.10 \\
4.31 \\
4.35 \\
1.42 \\
1.71 \\
3.81\end{array}$ & $\begin{array}{l}1.21 \\
0.19 \\
1.02 \\
0.81 \\
1.43 \\
1.61 \\
0.19\end{array}$ & $\begin{array}{r}17.50 \\
50.00 \\
30.00 \\
18.65 \\
5.56 \\
5.56 \\
50.00\end{array}$ & $\begin{array}{r}70 \\
233 \\
60 \\
15 \\
19 \\
8 \\
406\end{array}$ \\
\hline \multicolumn{7}{|c|}{ Table $18 \mathrm{c}$} \\
\hline \multicolumn{7}{|c|}{$\begin{array}{c}\text { Variation in Chloride/Sodium Ratio } \\
\text { by Precipitation Type }\end{array}$} \\
\hline type & wt. mean & mean & S. D. & $\underline{\min }$. & $\underline{\max }$. & $\underline{\mathrm{n}}$ \\
\hline $\begin{array}{l}\text { rain } \\
\text { rain shower } \\
\text { thundershower } \\
\text { snow } \\
\text { al1 }\end{array}$ & $\begin{array}{l}3.77 \\
2.39 \\
4.04 \\
4.12 \\
3.76\end{array}$ & $\begin{array}{l}3.49 \\
3.85 \\
3.60 \\
3.62 \\
3.55\end{array}$ & $\begin{array}{l}3.69 \\
4.90 \\
4.15 \\
3.06 \\
3.81\end{array}$ & $\begin{array}{l}0.81 \\
1.02 \\
1.11 \\
0.19 \\
0.19\end{array}$ & $\begin{array}{l}50.00 \\
30.00 \\
25.50 \\
18.65 \\
50.00\end{array}$ & $\begin{array}{r}289 \\
43 \\
34 \\
40 \\
406\end{array}$ \\
\hline \multicolumn{7}{|c|}{ Table 18d } \\
\hline ; & $\begin{array}{r}\text { Variation } \\
\text { by }\end{array}$ & $\begin{array}{l}\text { in Chlor } \\
\text { Precipit }\end{array}$ & $\begin{array}{l}\text { de/Sodium } \\
\text { tion Rate }\end{array}$ & Ratio & & \\
\hline rate (in./hour) & wt. mean & mean & S.D. & min. & $\underline{\max }$. & $\mathrm{n}$ \\
\hline $\begin{array}{l}0.00-0.03 \\
0.04-0.10 \\
0.11=0.25 \\
0.26-0.50 \\
\geq 0.51 \\
\text { al1 }\end{array}$ & $\begin{array}{l}3.60 \\
3.11 \\
4.54 \\
3.97 \\
3.45 \\
3.76\end{array}$ & $\begin{array}{l}3.64 \\
3.15 \\
4.40 \\
3.86 \\
3.11 \\
3.55\end{array}$ & $\begin{array}{l}3.63 \\
2.32 \\
7.03 \\
2.16 \\
1.43 \\
3.81\end{array}$ & $\begin{array}{l}0.81 \\
0.19 \\
1.29 \\
1.44 \\
1.43 \\
0.19\end{array}$ & $\begin{array}{r}30.00 \\
18.65 \\
50.00 \\
8.62 \\
5.56 \\
50.00\end{array}$ & $\begin{array}{r}151 \\
165 \\
58 \\
23 \\
9 \\
406\end{array}$ \\
\hline
\end{tabular}


wind speed

$(\mathrm{m} \mathrm{sec}-1)$

$0-2$

$3-5$

6-9

$\geq 10$

a11

Table 18e

Variation in Chloride/Sodium Ratio

by Wind Speed at $108 \mathrm{~m}$

\begin{tabular}{|c|c|c|c|c|c|}
\hline wt. mean & mean & S. D. & $\min$. & $\max$. & $\mathrm{n}$ \\
\hline 2.71 & 3.70 & 2.51 & 1.40 & 9.00 & 19 \\
\hline 4.98 & 4.34 & 4.99 & 0.19 & 50.00 & 154 \\
\hline 3.22 & 3.32 & 3.09 & 1.07 & 30.00 & 150 \\
\hline 2.77 & 2.47 & 2.01 & 0.81 & 18.65 & 83 \\
\hline 3.76 & 3.55 & 3.81 & 0.19 & 50.00 & 406 \\
\hline
\end{tabular}

Table $18 f$

Variation in Chloride/Sodium Ratio

by Wind Direction

direction

$\mathrm{N}$

NE

E

SE

$S$

SW

W

NW

a11

\begin{tabular}{c} 
wt. mean \\
\hline 2.73 \\
6.87 \\
3.89 \\
4.00 \\
2.46 \\
2.90 \\
4.31 \\
2.92 \\
3.76
\end{tabular}

\begin{tabular}{lll} 
mean & & S. D. \\
\cline { 1 - 1 } 2.72 & & 1.01 \\
4.28 & & 6.85 \\
3.69 & & 3.44 \\
3.34 & 2.42 \\
2.76 & 2.70 \\
3.06 & 1.48 \\
7.12 & 7.37 \\
3.49 & 2.82 \\
3.55 & 3.81
\end{tabular}

Table 18g

Variation in Chloride/Sodium Ratio

by Temperature

\begin{tabular}{l} 
temperature $\left({ }^{\circ} \mathrm{F}\right)$ \\
\hline $20-29$ \\
$30-39$ \\
$40-49$ \\
$50-59$ \\
$60-69$ \\
$70-79$ \\
$80-89$ \\
al1
\end{tabular}

\begin{tabular}{c} 
wt. mean \\
\hline 2.98 \\
2.93 \\
4.02 \\
3.49 \\
4.31 \\
3.74 \\
3.33
\end{tabular}

3.76

mean
2.86
2.90
3.73
3.87
3.95
3.19
3.33
3.55

\begin{tabular}{l} 
S. D. \\
\hline 1.56 \\
2.56 \\
5.61 \\
3.11 \\
3.57 \\
2.22 \\
- \\
3.81
\end{tabular}

min.

0.19

0.81

1.02

1.38

1.29

1.28

3.33

0.19 \begin{tabular}{r} 
max. \\
\hline 5.71 \\
50.00 \\
25.50 \\
13.57 \\
20.83 \\
6.30 \\
30.00 \\
18.18 \\
50.00
\end{tabular}

\begin{tabular}{r}
$\mathrm{n}$ \\
\hline 32 \\
50 \\
75 \\
75 \\
60 \\
47 \\
19 \\
48 \\
406
\end{tabular}

